\title{
Some prime factorization results for free quantum group factors
}

\author{
Yusuke Isono
}

\begin{abstract}
We prove some unique factorization results for tensor products of free quantum group factors. They are type III analogues of factorization results for direct products of bi-exact groups established by Ozawa and Popa. In the proof, we first take continuous cores of the tensor products, which satisfy a condition similar to condition (AO), and discuss some factorization properties for the continuous cores. We then deduce factorization properties for the original type III factors. We also prove some unique factorization results for crossed product von Neumann algebras by direct products of bi-exact groups.
\end{abstract}

\section{Introduction}

We say a $\mathrm{II}_{1}$ factor is prime if it is not isomorphic to tensor products of $\mathrm{II}_{1}$ factors. The first example of such a factor was given by Popa [19. He proved that any free group factor $L \mathbb{F}_{\infty}$ (with uncountably many generators) is prime. In [7, Ge proved that $L \mathbb{F}_{n}$ (with $n \geq 2$ ) are prime by computing Voiculescu's free entropy. Ozawa then proved that all free group factors are solid [14, meaning that the relative commutant of any diffuse von Neumann subalgebra is amenable (namely, injective). Solidity immediately yields primeness of any diffuse non-amenable subalgebras. Ozawa's proof relied on the notion of condition (AO) (see Subsection 2.1) and $C^{*}$-algebraic methods. Peterson gave a new proof of solidity of free group factors [16].

In [7, Ge asked the following question:

- Is $L \mathbb{F}_{2} \bar{\otimes} L \mathbb{F}_{2}$ isomorphic to $L \mathbb{F}_{2} \bar{\otimes} L \mathbb{F}_{2} \bar{\otimes} L \mathbb{F}_{2}$ ?

Here the symbol $\bar{\otimes}$ means the tensor product as von Neumann algebras. This is an extended primness problem for free group factors, which mentions numbers of tensor components. The question was solved by Ozawa and Popa [15]. They used a combination of a tensor product analogue of condition (AO) and Popa's intertwining techniques, and obtained a relative version of Ozawa's solidity theorem. As a result, they deduced the following theorem, which gave a complete answer to the problem. See [2, Section 15] for bi-exactness below.

Factorization theorem of Ozawa and Popa. Let $\Gamma_{i}(i=1, \ldots, m)$ be non-amenable, ICC, bi-exact discrete groups and $N_{j}(j=1, \ldots, n)$ be $\mathrm{II}_{1}$ factors. If $L \Gamma_{1} \bar{\otimes} \cdots \bar{\otimes} L \Gamma_{m}=$ $N_{1} \bar{\otimes} \cdots \bar{\otimes} N_{n}(=: M)$ and $m \leq n$, then $m=n$ and there are $u \in \mathcal{U}(M), \sigma \in \mathfrak{S}_{n}$, and $t_{i}>0$ with $t_{1} \cdots t_{n}=1$ such that $u N_{\sigma(i)} u^{*}=L \Gamma_{i}^{t_{i}}$ for a fixed decomposition $M=$ $L \Gamma_{1}^{t_{1}} \bar{\otimes} \cdots \bar{\otimes} L \Gamma_{n}^{t_{n}}$.

Here recall that for a $\mathrm{II}_{1}$ factor $M$ and $t>0$, the amplification $M^{t}$ is defined (up to *-isomorphism) as $p M p \bar{\otimes} \mathbb{M}_{n}$ for any $n \in \mathbb{N}$ with $t \leq n$ and any projection $p \in M$ with trace $t / n$. We also recall that $\mathrm{II}_{1}$ factors $M$ and $N$ are stably isomorphic if $M^{t} \simeq N^{s}$ for 
some $t, s>0$. For any $\mathrm{II}_{1}$ factors $M_{i}$ and $t>0, M_{1} \bar{\otimes} M_{2} \simeq\left(p M_{1} p \bar{\otimes} q M_{2} q\right) \bar{\otimes} \mathbb{M}_{n} \bar{\otimes} \mathbb{M}_{m} \simeq$ $M_{1}^{t} \bar{\otimes} M_{2}^{1 / t}$ for some large $n, m$ and projections $p, q$ with traces $t / n$ and $1 / m t$. So any $\mathrm{II}_{1}$ factor tensor decomposition is determined up to amplifications of tensor components. The theorem above then means the uniqueness of the tensor decomposition up to stable isomorphism.

In the present paper, we study similar factorization results for free quantum group factors. It is known that these factors satisfy condition (AO) [28] [30] [29], and in fact tensor products of these factors satisfy an analogue of condition (AO) mentioned above for free group factors (see Proposition 3.1.2). So Ozawa-Popa's factorization result is true if each tensor component is a non-amenable $\mathrm{II}_{1}$ factor. However these factors often become type III and, in the general case, Popa's techniques are no loner available.

To avoid the difficulty, we take continuous cores. A condition (AO) type phenomenon on cores of these factors was already observed in [10], and we generalize it to cores of the tensor products. This enables us to discuss some factorization properties on the continuous cores. In particular, we deduce some one-to-one correspondence with respect to Popa's embedding $\preceq$ (see Subsection 2.3) between tensor components on the cores. We then turn to see original type III algebras and deduce some factorization results. Thus we obtain the following theorem which is the main conclusion of the paper. See Definition 2.2.1 for the class $\mathcal{C}$, which contains (duals of) free quantum groups, and Subsection 2.1 for type $\mathrm{III}_{1}$ factors, Sd-invariants, continuous cores $C_{\phi}(N)$, and centralizer algebras $\left(N_{i}\right)_{\phi_{i}}$ below.

Theorem A. Let $\hat{\mathbb{G}}_{i}(i=1, \ldots, m)$ be discrete quantum groups in $\mathcal{C}$ and $N_{j}(j=1, \ldots, n)$ be non-amenable von Neumann algebras which admit almost periodic states. Assume that there is an inclusion $N:=N_{1} \bar{\otimes} \cdots \bar{\otimes} N_{n} \subset L^{\infty}\left(\mathbb{G}_{1}\right) \bar{\otimes} \cdots \bar{\otimes} L^{\infty}\left(\mathbb{G}_{m}\right)=: M$ with a faithful normal conditional expectation. Then we have $n \leq m$.

Assume further $n=m, N=M$, and the following conditions:

- Each $L^{\infty}\left(\mathbb{G}_{i}\right)$ is a factor of type $\mathrm{II}_{1}$ or $\mathrm{III}_{1}$ and its Haar state $h_{i}$ is $\operatorname{Sd}\left(L^{\infty}\left(\mathbb{G}_{i}\right)\right)$ almost periodic. Write $h:=h_{1} \otimes \cdots \otimes h_{n}$.

- Each $N_{i}$ is a factor of type $\mathrm{II}_{1}$ or $\mathrm{III}_{1}$ and any $\mathrm{III}_{1}$ factor $N_{i}$ admits an almost periodic state $\phi_{i}$ such that $\left(N_{i}\right)_{\phi_{i}}^{\prime} \cap N_{i}=\mathbb{C}$ (put the trace as $\phi_{i}$ when $N_{i}$ is a $\mathrm{II}_{1}$ factor). Write $\phi:=\phi_{1} \otimes \cdots \otimes \phi_{n}$.

Then under the canonical isomorphism $C_{\phi}(N) \simeq C_{h}(M)$ with the canonical trace Tr, there exists a unique $\sigma \in \mathfrak{S}_{n}$ such that

$$
p C_{\phi_{i}}\left(N_{i}\right) p \preceq_{C_{h}(M)} C_{\sigma(i)}\left(L^{\infty}\left(\mathbb{G}_{\sigma(i)}\right) \quad(i=1, \ldots, n)\right.
$$

for any projection $p \in L \mathbb{R} \subset C_{\phi}(N)$ with $\operatorname{Tr}(p)<\infty$. In this case, $N_{i}$ and $L^{\infty}\left(\mathbb{G}_{\sigma(i)}\right)$ are isomorphic when $N_{i}$ is a $\mathrm{III}_{1}$ factor, and stably isomorphic when $N_{i}$ is a $\mathrm{II}_{1}$ factor.

We mention that for any $\hat{\mathbb{G}} \in \mathcal{C}$, if $L^{\infty}(\mathbb{G})$ is a type $\mathrm{III}_{1}$ factor and its Haar state $h$ is $\operatorname{Sd}\left(L^{\infty}(\mathbb{G})\right.$ )-almost periodic, then it satisfies $L^{\infty}(\mathbb{G})_{h}^{\prime} \cap L^{\infty}(\mathbb{G})=\mathbb{C}$ (see Subsections 2.1 and 2.2). So as a particular case, we can put $N_{i}=L^{\infty}\left(\mathbb{H}_{i}\right)$ for $\hat{\mathbb{H}}_{i} \in \mathcal{C}$ with Haar state $\phi_{i}$ which is a trace or $\operatorname{Sd}\left(L^{\infty}\left(\mathbb{H}_{i}\right)\right)$-almost periodic.

In the paper, we also prove some unique factorization results for crossed product von Neumann algebras by direct product groups. In this situation, we assume that the given isomorphism preserves subalgebras on which groups act, so that we can compare direct product groups. We obtain the following theorem. In the theorem, the symbol $\rtimes$ means the crossed product von Neumann algebras. 
Theorem B. Let $\Gamma_{i}(i=1, \ldots, m)$ and $\Lambda_{j}(j=1, \ldots, n)$ be non-amenable discrete groups. Let $\left(A, \operatorname{Tr}_{A}\right)$ and $\left(B, \tau_{B}\right)$ be semifinite tracial von Neumann algebras with $\operatorname{Tr}_{A} \mid \mathcal{Z}(A)$ semifinite and $\tau_{B}(1)=1$. Write $\Gamma:=\Gamma_{1} \times \cdots \times \Gamma_{m}$ and $\Lambda:=\Lambda_{1} \times \cdots \times \Lambda_{n}$. Let $\alpha$ and $\beta$ be trace preserving actions of $\Gamma$ and $\Lambda$ on $\left(A, \operatorname{Tr}_{A}\right)$ and $\left(B, \tau_{B}\right)$ respectively. Assume the following conditions:

- There is an inclusion $B \rtimes \Lambda \subset p(A \rtimes \Gamma) p$ for a $\operatorname{Tr}_{A}$-finite projection $p \in \mathcal{Z}(A)$, which sends $B$ onto $p A p$.

- Either that $\mathcal{Z}(A)$ is diffuse or $A$ is a $\mathrm{II}_{1}$ factor.

- Actions $\alpha$ and $\beta$ are free on $A$ and $B$ respectively. Actions $\left.\alpha\right|_{\Gamma_{i}}$ and $\left.\beta\right|_{\Lambda_{j}}$ are ergodic on $\mathcal{Z}(A)$ and $\mathcal{Z}(B)$ respectively for all $i$ and $j$.

- All $\Gamma_{i}$ are bi-exact and $A$ is amenable.

Then we have $n \leq m$. If moreover $n=m$, then there exists a unique $\sigma \in \mathfrak{S}_{n}$ such that

$$
B \rtimes \Lambda_{i} \preceq_{A \rtimes \Gamma} A \rtimes \Gamma_{\sigma(i)} \quad(i=1, \ldots, n) .
$$

The same conclusions are true without amenability of $A$, if $\Gamma$ is weakly amenable, $\operatorname{Tr}_{A}$ is finite and $p=1_{A}$.

We mention that for $B \rtimes \Lambda_{i} \preceq_{A \rtimes \Gamma} A \rtimes \Gamma_{\sigma(i)}$, we can find an embedding and an intertwiner of a special form, which is discussed in Subsection 4.2 .

In the final section, we give a different approach to factorization properties for bi-exact and weakly amenable group factors.

Throughout the paper, we always assume that discrete groups are countable, quantum group $C^{*}$-algebras are separable, von Neumann algebras have separable predual, and Hilbert spaces are separable.

Acknowledgement. The author would like to thank Yuki Arano, Cyril Houdayer, Yasuyuki Kawahigashi, Narutaka Ozawa, Hiroki Sako, and Yoshimichi Ueda for fruitful conversations. He was supported by JSPS, Research Fellow of the Japan Society for the Promotion of Science.

\section{Preliminaries}

\subsection{Fullness and Discrete decompositions}

In the subsection, we recall Connes's discrete decomposition and related notions. We refer the reader to [4] (see also [6]).

Let $\omega$ be a free ultra filter on $\mathbb{N}$. Consider two $C^{*}$-algebras

$$
\begin{aligned}
A_{\omega} & :=\left\{\left(x_{n}\right)_{n} \in \ell^{\infty}(M) \mid\left\|\phi\left(\cdot x_{n}\right)-\phi\left(x_{n} \cdot\right)\right\|_{M_{*}} \rightarrow 0 \text { as } n \rightarrow \omega, \text { for all } \phi \in M_{*}\right\}, \\
J_{\omega} & :=\left\{\left(x_{n}\right)_{n} \in \ell^{\infty}(M) \mid x_{n} \rightarrow 0 \text { as } n \rightarrow \omega \text { in the } * \text {-strong topology }\right\} .
\end{aligned}
$$

The quotient $C^{*}$-algebra $A_{\omega} / J_{\omega}$ becomes a von Neumann algebra and we denote it by $M_{\omega}$. We say a factor $M$ is full if $\operatorname{Inn}(M)$ is closed in $\operatorname{Aut}(M)$ in the u-topology, namely, the topology of pointwise norm convergence in $M_{*}$. A factor $M$ is full if and only if $M_{\omega} \simeq \mathbb{C}$ for some (any) ultra filter $\omega$.

Let $M$ be a von Neumann algebra and $\phi$ a faithful normal semifinite weight on $M$. Then the modular operator $\Delta_{\phi}$ and the modular conjugation $J_{\phi}$ are defined on $L^{2}(M, \phi)$ 
as a closed operator and an anti linear map. The map $J_{\phi}$ satisfies $M^{\prime}=J_{\phi} M J_{\phi} \simeq M^{\text {op }}$ and it gives a canonical right action of $M^{\mathrm{op}}$ on $L^{2}(M, \phi)$. The family $\sigma_{t}^{\phi}:=\operatorname{Ad} \Delta_{\phi}^{i t}(t \in \mathbb{R})$ gives an $\mathbb{R}$-action on $M$ called the modular action of $\phi$. The continuous core is defined as $C_{\phi}(M):=M \rtimes_{\sigma^{\phi}} \mathbb{R}$, which does not depends on the choice of $\phi$. We say a type III factor $M$ is of type $\mathrm{III}_{1}$ if $C_{\phi}(M)$ is a $\mathrm{II}_{\infty}$ factor. The centralizer algebra is defined as

$$
M_{\phi}:=\left\{x \in M \mid \Delta_{\phi}^{i t} x=x \Delta_{\phi}^{i t} \text { for all } t \in \mathbb{R}\right\},
$$

We say the weight $\phi$ is almost periodic if the modular operator $\Delta_{\phi}$ is diagonalizable, namely, it is of the form $\Delta_{\phi}=\sum_{\lambda \in \operatorname{ptSp}\left(\Delta_{\phi}\right)} \lambda E_{\lambda}$, where $\operatorname{ptSp}\left(\Delta_{\phi}\right) \subset \mathbb{R}_{+}^{*}$ is the point spectrum of $\Delta_{\phi}$ and $E_{\lambda}$ are spectrum projections. For a subgroup $\Lambda \subset \mathbb{R}_{+}^{*}$, we say $\phi$ is $\Lambda$-almost periodic if it is almost periodic and $\operatorname{ptSp}\left(\Delta_{\phi}\right) \subset \Lambda$. Any almost periodic weight $\phi$ is semifinite on $M_{\phi}$ and hence there is a faithful normal conditional expectation from $M$ onto $M_{\phi}$ [23, Theorem IX.4.2]. When $M$ is a factor with an almost periodic weight, its $S d$-invariant is defined as

$$
\operatorname{Sd}(M):=\bigcap_{\phi \text { is almost periodic on } M} \operatorname{ptSp}\left(\Delta_{\phi}\right) .
$$

It becomes a subgroup of $\mathbb{R}_{+}^{*}$. When $M$ is a full type III factor, an almost periodic wight $\phi$ is $\operatorname{Sd}(M)$-almost periodic if and only if $\left(M_{\phi}\right)^{\prime} \cap M=\mathbb{C}$.

Recall from [14] that a von Neumann algebra $M \subset \mathbb{B}(H)$ satisfies condition (AO) if there are $\sigma$-weakly dense $C^{*}$-subalgebras $A \subset M$ and $B \subset M^{\prime}$ such that $A$ is locally reflexive and the map $A \otimes_{\text {alg }} B \ni a \otimes b \mapsto a b \in \mathbb{B}(H) / \mathbb{K}(H)$ is bounded with respect to the minimal tensor norm. The following lemma is well known.

Lemma 2.1.1. Let $M$ be a von Neumann algebra. If $M$ is non-amenable and satisfies condition (AO) in $\mathbb{B}\left(L^{2}(M)\right)$, then $C^{*}\left\{M, M^{\prime}\right\} \cap \mathbb{K}\left(L^{2}(M)\right) \neq 0$.

Proof. Write $\mathbb{K}:=\mathbb{K}\left(L^{2}(M)\right)$. Let $A \subset M$ and $B \subset M^{\prime}$ be $\sigma$-weakly dense unital $C^{*}$-subalgebras satisfying that the map $\nu: A \otimes_{\text {alg }} B \ni a \otimes b \mapsto a b \in \mathbb{B}\left(L^{2}(M)\right) / \mathbb{K}$ is bounded on $A \otimes_{\min } B$. If $C^{*}\left\{M, M^{\prime}\right\} \cap \mathbb{K}=0$, then the image of $\nu$ is contained in $\left(C^{*}\left\{M, M^{\prime}\right\}+\mathbb{K}\right) / \mathbb{K} \simeq C^{*}\left\{M, M^{\prime}\right\} /\left(C^{*}\left\{M, M^{\prime}\right\} \cap \mathbb{K}\right) \simeq C^{*}\left\{M, M^{\prime}\right\}$. Hence $\nu$ is bounded without the quotient of $\mathbb{K}$. Since $A$ is locally reflexive, $A$ is unital, and $\nu$ is normal on $A \otimes_{\min } \mathbb{C}$, we can extend $\nu$ on $M \otimes_{\min } B$. We again extend $\nu$ on $\mathbb{B}\left(L^{2}(M)\right) \otimes_{\min } B$ by Arveson's theorem and denote by $\Phi$. The restriction of $\Phi$ on $\mathbb{B}\left(L^{2}(M)\right) \otimes_{\min } \mathbb{C}$ is a conditional expectation onto $B^{\prime}=M$ (since $\mathbb{C} \otimes_{\min } B$ is contained in the multiplicative domain of $\Phi)$. Thus $M$ is amenable.

The following lemma is a general version of [5, Corollary 2.3]. We thank Cyril Houdayer for demonstrating the proof of the lemma.

Lemma 2.1.2. Let $M$ and $N$ be factors. If $C^{*}\left\{M, M^{\prime}\right\} \cap \mathbb{K}\left(L^{2}(M)\right) \neq 0$, then the map $N_{\omega} \ni\left(x_{n}\right)_{n} \mapsto\left(1 \otimes x_{n}\right)_{n} \in(M \bar{\otimes} N)_{\omega}$ is surjective. In particular, $M \bar{\otimes} N$ is full if $N$ is full (possibly $N=\mathbb{C}$ ).

Proof. Since $M$ is a factor, we have $C^{*}\left\{M, M^{\prime}\right\}^{\prime \prime}=\left(M \cap M^{\prime}\right)^{\prime}=\mathbb{B}\left(L^{2}(M)\right)$. Let $x \in$ $C^{*}\left\{M, M^{\prime}\right\} \cap \mathbb{K}\left(L^{2}(M)\right)$ and $y \in \mathbb{B}\left(L^{2}(M)\right)$ be non-zero elements. Let $y_{i} \in C^{*}\left\{M, M^{\prime}\right\}$ be a bounded net converging to $y$ strongly. Then the net $y_{i} x$ converges to $y x$ in the norm topology and hence we have $y x \in C^{*}\left\{M, M^{\prime}\right\}$. This implies $\mathbb{B}\left(L^{2}(M)\right) x \mathbb{B}\left(L^{2}(M)\right) \subset$ $C^{*}\left\{M, M^{\prime}\right\}$ and hence $\mathbb{K}\left(L^{2}(M)\right) \subset C^{*}\left\{M, M^{\prime}\right\}$.

Let $\phi$ and $\psi$ be faithful normal states on $M$ and $N$ respectively and write $H:=$ $L^{2}(M, \phi) \otimes L^{2}(N, \psi)$, where the symbol $\otimes$ means the tensor product of Hilbert spaces. Let 
$\left(x_{n}\right)_{n} \in(M \bar{\otimes} N)_{\omega}$, namely, $\left(x_{n}\right)_{n}$ be a bounded sequence satisfying $\lim _{n \rightarrow \omega}\left\|\left[x_{n}, \chi\right]\right\|_{(M \bar{\otimes} N)_{*}}=$ 0 for any $\chi \in(M \bar{\otimes} N)_{*}$. We will show $x_{n}-\left(\phi \otimes \operatorname{id}_{N}\right)\left(x_{n}\right) \rightarrow 0$ as $n \rightarrow \omega$ in the $*$-strong topology, which means $\left(x_{n}\right)_{n}=\left(\left(\phi \otimes \mathrm{id}_{N}\right)\left(x_{n}\right)\right)_{n} \in N_{\omega}$. Since $\left[x_{n}, b a\right]=b\left[x_{n}, a\right] \rightarrow 0$ *-strongly for $a \in M \otimes_{\min } \mathbb{C}$ and $b \in M^{\prime} \otimes_{\min } \mathbb{C}$, we have $\left[x_{n}, a\right] \rightarrow 0$ for any $a \in$ $C^{*}\left\{M, M^{\prime}\right\} \otimes_{\min } \mathbb{C}$. Let $P_{a, 1}$ be the partial isometry from $\mathbb{C} \hat{1}$ to $\mathbb{C} \hat{a}$ for $a \in M$, which is contained in $\mathbb{K}\left(L^{2}(M)\right) \subset C^{*}\left\{M, M^{\prime}\right\}$. Then for any $a \in M$ and $b \in N$, we have

$$
\begin{aligned}
\left(x_{n}-\left(\phi \otimes \mathrm{id}_{N}\right)\left(x_{n}\right)\right)(\hat{a} \otimes \hat{b}) & \left.=x_{n}\left(P_{a, 1} \otimes 1\right)(\hat{1} \otimes \hat{b})-\left(P_{a, 1} \otimes 1\right)\left(\phi \otimes \mathrm{id}_{N}\right)\left(x_{n}\right)\right)(\hat{1} \otimes \hat{b}) \\
& =x_{n}\left(P_{a, 1} \otimes 1\right)(\hat{1} \otimes \hat{b})-\left(P_{a, 1} \otimes 1\right) x_{n}(\hat{1} \otimes \hat{b}) \\
& =\left[x_{n},\left(P_{a, 1} \otimes 1\right)\right](\hat{1} \otimes \hat{b}) \rightarrow 0
\end{aligned}
$$

in the norm topology of $H$. Since the same is true for $x_{n}^{*}$, we have $x_{n}-\left(\phi \otimes \mathrm{id}_{N}\right)\left(x_{n}\right) \rightarrow 0$ in the $*$-strong topology.

Lemma 2.1.3. Let $M_{i}$ be full factors with $C^{*}\left\{M_{i}, M_{i}^{\prime}\right\} \cap \mathbb{K}\left(L^{2}\left(M_{i}\right)\right) \neq 0$. Let $\phi_{i}$ be faithful normal semifinite weight on $M_{i}$ which is $\operatorname{Sd}\left(M_{i}\right)$-almost periodic. Then $\phi_{1} \otimes \cdots \otimes \phi_{n}$ is $\operatorname{Sd}\left(M_{1} \bar{\otimes} \cdots \bar{\otimes} M_{n}\right)$-almost periodic. In particular $\operatorname{Sd}\left(M_{1} \bar{\otimes} \cdots \bar{\otimes} M_{n}\right)=\operatorname{Sd}\left(M_{1}\right) \cdots \operatorname{Sd}\left(M_{n}\right)$ and $\left(M_{1} \bar{\otimes} \cdots \bar{\otimes} M_{n}\right)_{\phi_{1} \otimes \cdots \otimes \phi_{n}}$ is a factor.

Proof. By the previous lemma, $M_{1} \bar{\otimes} \cdots \bar{\otimes} M_{n}$ is a full factor. We have

$$
\begin{aligned}
& \left(M_{1} \bar{\otimes} \cdots \bar{\otimes} M_{n}\right)_{\phi_{1} \otimes \cdots \otimes \phi_{n}}^{\prime} \cap\left(M_{1} \bar{\otimes} \cdots \bar{\otimes} M_{n}\right) \\
\subset & \left(\left(M_{1}\right)_{\phi_{1}} \bar{\otimes} \cdots \bar{\otimes}\left(M_{n}\right)_{\phi_{n}}\right)^{\prime} \cap\left(M_{1} \bar{\otimes} \cdots \bar{\otimes} M_{n}\right) \\
= & \left(M_{1} \cap\left(M_{1}\right)_{\phi_{1}}^{\prime}\right) \bar{\otimes} \cdots \bar{\otimes}\left(M_{n} \cap\left(M_{n}\right)_{\phi_{n}}\right)^{\prime}=\mathbb{C} .
\end{aligned}
$$

Next we recall discrete decompositions. Let $M$ be a von Neumann algebra and $\phi$ a $\Lambda$-almost periodic wight on $M$ for a countable subgroup $\Lambda \subset \mathbb{R}_{+}^{*}$. As a discrete group, take the Pontryagin dual of $\Lambda$ and denote by $K$. By definition, we have a map $\mathbb{R} \simeq \widehat{\mathbb{R}_{+}^{*}} \rightarrow K$, which is injective and has a dense image if $\Lambda \subset \mathbb{R}_{+}^{*}$ is dense, and which is surjective if $\Lambda \subset \mathbb{R}_{+}^{*}$ is periodic. The modular action of $\mathbb{R}$ extends to an action of $K$. Take the crossed product von Neumann algebra $M \rtimes K$ by the action. Then by Takesaki duality, we have $(M \rtimes K) \rtimes \Lambda \simeq M \bar{\otimes} \mathbb{B}\left(\ell^{2}(\Lambda)\right)$. The dual weight of $M \bar{\otimes} \mathbb{B}\left(\ell^{2}(\Lambda)\right)$ is of the form $\phi \otimes \omega$, where $\omega:=\operatorname{Tr}(\lambda \cdot)$ for a closed operator $\lambda$ on $\ell^{2}(\Lambda)$ given by $\lambda(a)=a$. Since $M \rtimes K$ is the fixed point algebra of the dual action on $(M \rtimes K) \rtimes \Lambda$, we have $M \rtimes K \simeq\left(M \bar{\otimes} \mathbb{B}\left(\ell^{2}(\Lambda)\right)\right)_{\phi \otimes \omega}=: D_{\phi}(M)$. When $M$ is of type III, we have $D_{\phi}(M) \rtimes \Lambda \simeq M$, which is called a discrete decomposition. The subalgebra $D_{\phi}(M)$ is called a discrete core of M. Since $\phi \otimes \omega$ is almost periodic (or $\Lambda$ is discrete), there exists a faithful normal conditional expectation from $M$ onto $D_{\phi}(M)$. When $M$ is a full factor, there always exists an $\operatorname{Sd}(M)$-almost periodic weight $\phi$ on $M$. In this case, since $\phi \otimes \omega$ is $\operatorname{Sd}(M)$-almost periodic on $M \bar{\otimes} \mathbb{B}\left(\ell^{2}(\Lambda)\right) \simeq M$, the discrete core $D_{\phi}(M)=\left(M \bar{\otimes} \mathbb{B}\left(\ell^{2}(\Lambda)\right)\right)_{\phi \otimes \omega}$ is a $\mathrm{II}_{\infty}$ factor, and hence is identified as $M_{\phi} \bar{\otimes} \mathbb{B}(H)$ for some separable infinite Hilbert space $H$.

Finally $M$ is amenable if and only if $D_{\phi}(M)$ is amenable for any von Neumann algebra $M$ and its almost periodic weight $\phi$. Hence when $M$ is of type III and non-amenable, since $D_{\phi}(M) \simeq\left(M \bar{\otimes} \mathbb{B}\left(\ell^{2}(\Lambda)\right)\right)_{\phi \otimes \omega}$, there is an almost periodic weight on $M\left(\simeq M \bar{\otimes} \mathbb{B}\left(\ell^{2}(\Lambda)\right)\right)$ such that its centralizer algebra is non-amenable. By using a type decomposition, We have the same result for any von Neumann algebra with almost periodic weights. We write this observation as follows.

Lemma 2.1.4. Let $M$ be a non-amenable von Neumann algebra which admits an almost periodic wight. Then there exists an almost periodic weight $\phi$ such that $M_{\phi}$ is nonamenable. 


\subsection{Compact and discrete quantum groups}

Let $\mathbb{G}$ be a compact quantum group. In the paper, we use the following notation, which are same as in our previous works [10] 12. We denote the Haar state by $h$, the set of all equivalence classes of all irreducible unitary corepresentations by $\operatorname{Irred}(\mathbb{G})$, and right and left regular representations by $\rho$ and $\lambda$ respectively. For $x \in \operatorname{Irred}(\mathbb{G}),\left(u_{i, j}^{x}\right)_{i, j=1}^{n_{x}}$ are coefficients of $x$ and we frequently omit $n_{x}$. We regard $C_{\text {red }}(\mathbb{G}):=\rho(C(\mathbb{G}))$ as our main object. The GNS representation of $h$ is written as $L^{2}(\mathbb{G})$. All dual objects are written with hat (e.g. $\hat{\mathbb{G}})$.

Let $F$ be a matrix in $\mathrm{GL}(n, \mathbb{C})$. The free unitary quantum group (resp. free orthogonal quantum group) of $F\left[31\left[32\right.\right.$ is the $C^{*}$-algebra $C\left(A_{u}(F)\right.$ ) (resp. $C\left(A_{o}(F)\right)$ ) defined as the universal unital $C^{*}$-algebra generated by all the entries of a unitary $n$ by $n$ matrix $u=\left(u_{i, j}\right)_{i, j}$ satisfying that $F\left(u_{i, j}^{*}\right)_{i, j} F^{-1}$ is unitary (resp. $F\left(u_{i, j}^{*}\right)_{i, j} F^{-1}=u$ ).

In [12, we observed for free quantum groups, there is a nuclear $C^{*}$-algebra

$$
\mathcal{C}_{l} \subset C^{*}\left\{C_{\mathrm{red}}(\mathbb{G}), \hat{\lambda}\left(\ell^{\infty}(\hat{\mathbb{G}})\right)\right\} \subset \mathbb{B}\left(L^{2}(\mathbb{G})\right)
$$

such that

(a) it contains $C_{\text {red }}(\mathbb{G})$ and $\mathbb{K}\left(L^{2}(\mathbb{G})\right)$;

(b) all commutators of $\mathcal{C}_{l}$ and $C_{\text {red }}(\mathbb{G})^{\text {op }}$ are contained in $\mathbb{K}\left(L^{2}(\mathbb{G})\right)$, where $C_{\text {red }}(\mathbb{G})^{\text {op }}$ acts on $L^{2}(\mathbb{G})$ canonically.

When we see it in the continuous core $L^{\infty}(\mathbb{G}) \rtimes \mathbb{R} \subset \mathbb{B}\left(L^{2}(\mathbb{G}) \otimes L^{2}(\mathbb{R})\right.$ ) (with respect to the Haar state $h$ ), it also satisfies:

(c) a family of maps $\operatorname{Ad} \Delta_{h}^{i t}(t \in \mathbb{R})$ gives a norm continuous action of $\mathbb{R}$ on $\mathcal{C}_{l}$;

(d) all commutators of $\pi\left(\mathcal{C}_{l}\right)$ and $C_{\text {red }}(\mathbb{G})^{\text {op }} \otimes_{\text {min }} 1$ are contained in $\mathbb{K}\left(L^{2}(\mathbb{G})\right) \otimes_{\text {min }}$ $\mathbb{B}\left(L^{2}(\mathbb{R})\right)$.

Here $\pi$ means the canonical $*$-homomorphism from $\mathbb{B}\left(L^{2}(\mathbb{G})\right)$ into $\mathbb{B}\left(L^{2}(\mathbb{G}) \otimes L^{2}(\mathbb{R})\right)$ defined by $(\pi(x) \xi)(t):=\Delta_{h}^{-i t} x \Delta_{h}^{i t} \xi(t)$ for $x \in \mathbb{B}\left(L^{2}(\mathbb{G})\right), t \in \mathbb{R}$, and $\xi \in L^{2}(\mathbb{G}) \otimes L^{2}(\mathbb{R})$.

In the paper, our essential assumptions on quantum groups are these four conditions and we actually treat quantum subgroups at the same time. We are only interested in non-amenable von Neumann algebras, since amenable one is not prime. So we use the following terminology.

Definition 2.2.1. Let $\hat{\mathbb{G}}$ be a discrete quantum group. We say $\hat{\mathbb{G}}$ is in $\mathcal{C}$ if $L^{\infty}(\mathbb{G})$ is non-amenable and there exists a discrete quantum group $\hat{\mathbb{H}}$ such that

- the quantum group $\hat{\mathbb{G}}$ is a quantum subgroup of $\hat{\mathbb{H}}$;

- there exists a nuclear $C^{*}$-algebra $\mathcal{C}_{l} \subset C^{*}\left\{C_{\text {red }}(\mathbb{H}), \hat{\lambda}\left(\ell^{\infty}(\hat{\mathbb{H}})\right)\right\} \subset \mathbb{B}\left(L^{2}(\mathbb{H})\right)$ which satisfies conditions from (a) to (d) for $\hat{\mathbb{H}}$.

In our previous work, we already found following examples (see Subsection 3.2 and the proof of Theorem $\mathrm{C}$ in [12]).

Proposition 2.2.2. Let $\mathbb{G}$ be one of the following quantum groups.

(i) A co-amenable compact quantum group.

(ii) The free unitary quantum group $A_{u}(F)$ for any $F \in \mathrm{GL}(n, \mathbb{C})$. 
(iii) The free orthogonal quantum group $A_{o}(F)$ for any $F \in \mathrm{GL}(n, \mathbb{C})$.

(iv) The quantum automorphism group $A_{\text {aut }}(B, \phi)$ for any finite dimensional $C^{*}$-algebra $B$ and any faithful state $\phi$ on $B$.

(v) The dual of a bi-exact discrete group $\Gamma$.

(vi) The dual of a free product $\hat{\mathbb{G}}_{1} * \cdots * \hat{\mathbb{G}}_{n}$, where each $\mathbb{G}_{i}$ is as in from (i) to (v) above. Then the dual $\hat{\mathbb{G}}$ is in $\mathcal{C}$ if $L^{\infty}(\mathbb{G})$ is non-amenable.

Since conditions (a) and (b) above implies bi-exactness [12, Lemmas 3.1.4 and 3.3.1], which obviously implies condition (AO), we easily deduce the following lemma.

Lemma 2.2.3. Let $\hat{\mathbb{G}}$ be in $\mathcal{C}$. Then we have $C^{*}\left\{L^{\infty}(\mathbb{G}), L^{\infty}(\mathbb{G})^{\prime}\right\} \cap \mathbb{K}\left(L^{2}(\mathbb{G})\right) \neq 0$.

Let $\mathbb{G}$ be a compact quantum groups and $h$ its Haar state. Let $\left(u_{i, j}^{x}\right)_{i, j}^{x}$ be a fixed basis of the dense Hopf *-algebra $\mathcal{A}$ of $C_{\text {red }}(\mathbb{G})$. Assume that they are orthogonal in $L^{2}(\mathbb{G})$. In this case, the modular action of the Haar state $h$ satisfies $\sigma_{t}^{h}\left(u_{i, j}^{x}\right)=\left(\lambda_{i}^{x} \lambda_{j}^{x}\right)^{i t} u_{i, j}^{x}$ for some scalars $\lambda_{i}^{x}$. Let $E_{h}$ be the $h$-preserving conditional expectation from $L^{\infty}(\mathbb{G})$ onto $L^{\infty}(\mathbb{G})_{h}$ which extends to the projection $e_{h}$ from $L^{2}(\mathbb{G})$ onto $L^{2}\left(L^{\infty}(\mathbb{G})_{h}\right)$. Let $\mathcal{A}_{h} \subset L^{\infty}(\mathbb{G})_{h}$ be all the linear spans of $\left\{u_{i, j}^{x} \mid \lambda_{i}^{x} \lambda_{j}^{x}=1\right\}$, which is a $*$-algebra, and $C_{\text {red }}(\mathbb{G})_{h}$ be its norm closure. For $a \in L^{\infty}(\mathbb{G})_{h}$ with the Fourier expansion $a=\sum_{x \in \operatorname{Irred}(\mathbb{G}), i, j} a_{i, j}^{x} u_{i, j}^{x}$, we have $a=\sigma_{t}^{h}(a)=\sum_{x \in \operatorname{Irred}(\mathbb{G}), i, j} a_{i, j}^{x}\left(\lambda_{i}^{x} \lambda_{j}^{x}\right)^{i t} u_{i, j}^{x}$ in $L^{2}(\mathbb{G})$ and hence $a_{i, j}^{x}=0$ if $\lambda_{i}^{x} \lambda_{j}^{x} \neq 1$. This means that $e_{h}$ is the projection onto the subspace spanned by $\mathcal{A}_{h}$, and hence we have $E_{h}\left(u_{i, j}^{x}\right)=u_{i, j}^{x}$ when $\lambda_{i}^{x} \lambda_{j}^{x}=1$, and $E_{h}\left(u_{i, j}^{x}\right)=0$ when $\lambda_{i}^{x} \lambda_{j}^{x} \neq 1$. Hence $E_{h}$ is a map from $\mathcal{A}$ onto $\mathcal{A}_{h}$ which is the identity on $\mathcal{A}_{h}$. This implies that $\mathcal{A}_{h}$ is $\sigma$-weakly dense in $L^{\infty}(\mathbb{G})_{h}$ and $E_{h}$ restricts to a conditional expectation from $C_{\text {red }}(\mathbb{G})$ onto $C_{\text {red }}(\mathbb{G})_{h}$.

\subsection{Popa's intertwining techniques}

In the paper, we use Popa's intertwining techniques for type III subalgebras, although there is no useful equivalent conditions in this case. So our definition here is more general than usual one.

Definition 2.3.1. Let $M$ be a von Neumann algebra, $p$ and $q$ projections in $M, A \subset p M p$ and $B \subset q M q$ von Neumann subalgebras. Assume that $B$ is finite or type III. We say $A$ embeds in $B$ inside $M$ and denote by $A \preceq_{M} B$ if there exist non-zero projections $e \in A$ and $f \in B$, a unital normal $*$-homomorphism $\theta: e A e \rightarrow f B f$, and a partial isometry $v \in M$ such that

- $v v^{*} \leq e$ and $v^{*} v \leq f$,

- $v \theta(x)=x v$ for any $x \in e A e$.

We first recall characterizations of this condition for non-finite and semifinite von Neumann algebras (with finite subalgebras).

Theorem 2.3.2 (non-finite version, [18] [17] 25] 9]). Let $M, p, q, A$, and $B$ be as in the definition above and let $E_{B}$ be a faithful normal conditional expectation from $q M q$ onto $B$. Assume that $B$ is finite with a trace $\tau_{B}$. Then the following conditions are equivalent.

(i) We have $A \preceq_{M} B$.

(ii) There exists no sequence $\left(w_{n}\right)_{n}$ of unitaries in $A$ such that $\left\|E_{B}\left(b^{*} w_{n} a\right)\right\|_{2, \tau_{B}} \rightarrow 0$ for any $a, b \in p M q$. 
(iii) There exists a non-zero $A$-B-submodule $H$ of $p L^{2}(M) q$ with $\operatorname{dim}_{\left(B, \tau_{B}\right)} H<\infty$.

Theorem 2.3.3 (semifinite version, 3] 8]). Let $M$ be a semifinite von Neumann algebra with a faithful normal semifinite trace $\operatorname{Tr}$, and $B \subset M$ be a von Neumann subalgebra with $\operatorname{Tr}_{B}:=\left.\operatorname{Tr}\right|_{B}$ semifinite. Denote by $E_{B}$ the unique Tr-preserving conditional expectation from $M$ onto $B$. Let $p$ be a Tr-finite projection in $M$ and $A \subset p M p$ a von Neumann subalgebra. Then the following conditions are equivalent.

(i) There exists a non-zero projection $p \in B$ with $\operatorname{Tr}_{B}(p)<\infty$ such that $A \preceq_{r M r} q B q$, where $r:=p \vee q$.

(ii) There exists no sequence $\left(w_{n}\right)_{n}$ in unitaries of $A$ such that $\left\|E_{B}\left(b^{*} w_{n} a\right)\right\|_{2, \operatorname{Tr}_{B}} \rightarrow 0$ for any $a, b \in p M$.

We use the same symbol $A \preceq_{M} B$ if one of these conditions holds.

Remark 2.3.4. By the proof, when $A \preceq_{M} B$ for semifinite $B \subset M$, for any increasing net $\left(q_{i}\right)_{i} \subset B$ of $\operatorname{Tr}_{B}$-finite projections, we can find some $q_{i}$ such that $A \preceq_{r M r} q_{i} B q_{i}$ where $r:=p \vee q_{i}$. So we can choose such a $q_{i}$ from any semifinite subalgebra of $B$ on which $\operatorname{Tr}_{B}$ is semifinite.

Since we mainly study continuous cores in the paper, the theorem for semifinite algebras are important for us. Theorem 2.3.3 is a generalization of the statement (ii) in Theorem 2.3.2 and this formulation is, for example, useful in the next subsection. However, to study our factorization properties, the statement (iii) is also important since the proof of Ozawa and Popa for prime factorization result relied on them. Hence here we give corresponding statements in the semifinite setting. We include sketches of proofs, since we use similar strategies later. See [2, Theorem F.12] for the details. Recall that any opposite von Neumann algebra $M^{\text {op }}$ has a canonical right action on $L^{2}(M)$, and we write its element as $a^{\text {op }} \in M^{\text {op }}$ for $a \in M$.

Proposition 2.3.5. Let $M, B, \operatorname{Tr}, p, A$, and $E_{B}$ be as in the previous theorem. Let $q \in B$ be a non-zero projection with $\operatorname{Tr}_{B}(q)<\infty$ and write as $z_{q}$ the central support projection of $q$ in $B$. Let $e_{B}$ be the Jones projection of $B \subset M$ and let $\operatorname{Tr}_{\langle M, B\rangle}$ be the semifinite trace on $\langle M, B\rangle$ given by $M e_{B} M \ni x e_{B} y \mapsto \operatorname{Tr}(x y)$. Then the following conditions are equivalent.

(i) We have $A \preceq_{r M r} q B q$ for $r:=p \vee q$.

(ii) There exists a non-zero positive element $d_{0} \in\langle M, B\rangle$ such that $d_{0} p z_{q}^{\mathrm{op}}=d_{0}, \operatorname{Tr}_{\langle M, B\rangle}\left(d_{0}\right)<$ $\infty$, and $K_{d_{0}}^{A} \not \supset 0$. Here $K_{d_{0}}^{A}$ is the $\sigma$-weak closure of $\operatorname{co}\left\{w d_{0} w^{*} \mid w \in \mathcal{U}(A)\right\}$.

(iii) There exists a non-zero positive element $d \in\langle M, B\rangle \cap A^{\prime}$ such that $d p z_{q}^{\mathrm{op}}=d$ and $\operatorname{Tr}_{\langle M, B\rangle}(d)<\infty$.

(iv) There exists a non-zero $p A p-q B q$-submodule $H$ of $p L^{2}(M, \operatorname{Tr}) q$ with $\operatorname{dim}_{\left(q B q, \tau_{q}\right)} H<$ $\infty$, where $\tau_{q}:=\operatorname{Tr}_{B}(q \cdot q) / \operatorname{Tr}_{B}(q)$.

We use the same symbol $A \preceq_{M} B$ if one of these conditions holds.

Proof. The equivalence of (i) and (iv) follows from Theorem 2.3.2, since we canonically have $p L^{2}(M, \operatorname{Tr}) q=p L^{2}(r M r, \operatorname{Tr}(r \cdot r)) q$. We show implications (i) $\Rightarrow$ (ii) $\Rightarrow$ (iii) $\Rightarrow$ (iv).

Suppose condition (i). Then by the theorem above, we can find $\delta>0$ and a finite subset $\mathcal{F} \subset p M q$ such that $\left\|E_{B}\left(y^{*} w x\right)\right\|_{2, \tau_{q}}>\delta$ for any $x, y \in \mathcal{F}$ and $w \in \mathcal{U}(A)$, where $\tau_{q}:=\operatorname{Tr}(q \cdot q) / \operatorname{Tr}(q)$. Put $d_{0}:=\sum_{x \in \mathcal{F}} x e_{B} x^{*} \in\langle M, B\rangle$. It obviously satisfies $d_{0}=d_{0} p$ 
and $\operatorname{Tr}_{\langle M, B\rangle}\left(d_{0}\right)<\infty$. We also have $d_{0} z_{q}^{\mathrm{op}}=d_{0}$ since $z_{q}^{\mathrm{op}} e_{B}=z_{q} e_{B}$. To see the condition on $K_{d_{0}}^{A}$, we calculate for any $w \in \mathcal{U}(\mathcal{A})$

$\sum_{x \in \mathcal{F}}\left\langle w^{*} d_{0} w \hat{x} \mid \hat{x}\right\rangle=\sum_{x, y \in \mathcal{F}}\left\langle e_{B} y^{*} w \hat{x} \mid y^{*} w \hat{x}\right\rangle=\sum_{x, y \in \mathcal{F}}\left\|e_{B} y^{*} w \hat{x}\right\|_{2, \tau_{q}}=\sum_{x, y \in \mathcal{F}}\left\|E_{B}\left(y^{*} w x\right)\right\|_{2, \tau_{q}}>\delta$.

Hence we get $K_{d_{0}}^{A} \not \supset 0$ and (ii) holds.

Next suppose condition (ii). We regard $K_{d_{0}}^{A} \subset\langle M, B\rangle$ as an $L^{2}$-norm bounded, convex, weak closed (and hence $L^{2}$-norm closed) subset of $L^{2}\left(\langle M, B\rangle, \operatorname{Tr}_{\langle M, B\rangle}\right)$. Then we can find the circumcenter $d$ of $K_{d_{0}}^{A}$, which is non-zero by assumption. By the uniqueness of the circumcenter, we have $w d w^{*}=d$ and hence $d$ is contained in $\langle M, B\rangle \cap A^{\prime}$. Since $d \in K_{d_{0}}^{A}$, we have $\operatorname{Tr}_{\langle M, B\rangle}(d)<\infty$ by the normality of $\operatorname{Tr}_{\langle M, B\rangle}$. Thus condition (iii) holds.

Suppose condition (iii). Take a non-zero spectral projection $f$ of $d$ such that $\operatorname{Tr}_{\langle M, B\rangle}(f)<$ $\infty$. Since $f$ is also contained in $\langle M, B\rangle \cap A^{\prime}$ with $f=f p z_{q}^{\text {op }}$, the closed subspace $H:=f q^{\mathrm{op}} L^{2}(M)$, which is nonzero since $f \leq z_{q}^{\mathrm{op}}$, has a $p A p-q B q$-bimodule structure as a submodule of $L^{2}(M)$. Now the dimension of $H$ with respect to $\left(q B q, \tau_{q}\right)$ is smaller than $\operatorname{Tr}_{\langle M, B\rangle}(f) / \operatorname{Tr}_{\langle M, B\rangle}(q)$ and hence $H$ has a finite dimension. We get condition (iv).

Corollary 2.3.6. Keep the setting in the previous proposition. Then $A \preceq_{M} B$ if and only if there exists a non-zero positive element $d \in p\langle M, B\rangle p \cap A^{\prime}$ such that $\operatorname{Tr}_{\langle M, B\rangle}(d)<\infty$. Also $A \preceq_{M} B$ if and only if $e A e \preceq_{r M r} f B f$ for any (some) projections $e \in A$ and $f \in B$ with $\operatorname{Tr}_{B}(f)<\infty$ and with central supports $1_{A}$ and $1_{B}$ respectively.

Proof. For the first half, the only if direction is trivial from the previous theorem. Let $\left(q_{i}\right) \subset B$ be an increasing net of $\operatorname{Tr}_{B}$-finite projections converging to $1_{B}$. Let $d$ be in the statement. Then there is some $i$ such that $\tilde{d}:=d z_{q_{i}}^{\mathrm{op}} \neq 0$. Then $\tilde{d}$ satisfies $\operatorname{Tr}_{\langle M, B\rangle}(\tilde{d})<\infty$ and $\tilde{d}=\tilde{d} z_{q_{i}}^{\mathrm{op}} p$. Since $z_{q_{i}}^{\mathrm{op}} \in \mathcal{Z}(\langle M, B\rangle) \subset\langle M, B\rangle \cap A^{\prime}$, we have $\tilde{d} \in\langle M, B\rangle \cap A^{\prime}$.

For the second half, suppose $A \preceq_{M} B$ and take $d$ in the first statement. If $d e=0$, then $d z_{A}(e)=d$ is also zero since $d \in A^{\prime}$. So $d e=d e z_{f}^{\mathrm{op}}$ is non-zero and we have $e A e \preceq_{r M r} f B f$.

Corollary 2.3.7. Keep the setting in the previous proposition and assume that $A$ is of type II. Then the following condition is also equivalent to $A \preceq_{r M r} q B q$.

(v) There exist non-zero projections $e \in A$ and $f \in q B q$, and a partial isometry $V \in$ $\langle M, B\rangle$ such that $V^{*} V \in e A^{\prime}$ and $V V^{*}=f e_{B}$, where $e_{B}$ is the Jones projection for $B \subset M$.

In the case, $e \in A$ is taken from any type II subalgebra of $A$.

Proof. Before the proof, we observe that there is an extended center valued trace $T$ on $z_{q}^{\mathrm{op}}\langle M, B\rangle$ such that $T\left(q e_{B}\right)=z_{q}^{\mathrm{op}}$ (for extended center valued traces, see the final part of [22, Subsection V.2]). Note that $z_{q}^{\mathrm{op}}$ is the central support of $q^{\mathrm{op}}$ in $B^{\mathrm{op}}$ and hence is contained in $\mathcal{Z}(\langle M, B\rangle)$.

Since the central support of $q e_{B}$ in $\langle M, B\rangle$ is $z_{q}^{\text {op }}$, we have an isomorphism

$$
\pi: z_{q}^{\mathrm{op}} \mathcal{Z}(\langle M, B\rangle)=\left(z_{q} \mathcal{Z}(B)\right)^{\mathrm{op}} \ni\left(z_{q} a\right)^{\mathrm{op}} \mapsto q e_{B}\left(z_{q} a\right)^{\mathrm{op}}=q e_{B} a \in q e_{B} \mathcal{Z}(B) .
$$

Put $\phi:=\operatorname{Tr}_{\langle M, B\rangle} \circ \pi$. Then $\phi$ is a faithful normal positive functional on $z_{q}^{\mathrm{op}} \mathcal{Z}(\langle M, B\rangle)$ and hence there is an extended center valued trace $T$ on $z_{q}^{\mathrm{op}}\langle M, B\rangle$ defined by the equation $\operatorname{Tr}_{\langle M, B\rangle}(x a)=\phi(T(x) a)$ for all $x \in z_{q}^{\mathrm{op}}\langle M, B\rangle^{+}$and $a \in z_{q}^{\mathrm{op}} \mathcal{Z}(\langle M, B\rangle)^{+}$. It holds that $T\left(q e_{B}\right)=z_{q}^{\mathrm{op}}$. 
Suppose condition (iii) in the previous proposition. Taking a spectral projection, we assume $d$ is a projection. Let $T$ be the extended center valued trace on $z_{q}^{\mathrm{op}}\langle M, B\rangle$ constructed above. Since $\operatorname{Tr}_{\langle M, B\rangle}(d)<\infty$, it is a finite projection and so it takes finite values almost everywhere as a function on the spectrum of $\mathcal{Z}(\langle M, B\rangle)$ (e.g. 22, Proposition V.2.35]). Hence there exists a projection $z \in \mathcal{Z}(\langle M, B\rangle)$ such that $T(z d) \leq n z_{q}^{\text {op }}$ for some $n \in \mathbb{N}$. Replacing $d$ with $z d$, we may assume $T(d) \leq n z_{q}^{\mathrm{op}}$. Since $A$ is of type II, there exist mutually orthogonal and equivalent projections $e_{i}$ such that $1_{A}=\sum_{i=1}^{n} e_{i}$. Then it is easy to show that $0 \neq T\left(d e_{1}\right) \leq z_{q}^{\text {op }}$. Write $e:=e_{1}$. Then $T(d e) \leq z_{q}^{\text {op }}$ implies $d e \prec q e_{B}$. There is a partial isometry $V \in z_{q}^{\mathrm{op}}\langle M, B\rangle$ such that $V^{*} V=d e$ and $V V^{*} \leq q e_{B}$. Writing $V V^{*}=f e_{B}$ for some $f \in q B q$, we are done. Note that $e_{i}$ above are taken from any type II subalgebra of $A$ and hence the final assertion also holds.

Next suppose condition (v). Since $V e A e V^{*} \subset f e_{B}\langle M, B\rangle f e_{B}=f B f e_{B} \simeq f B f$ and $V^{*} V$ commutes with $e A e$, we can define a unital normal $*$-homomorphism $\theta$ from $e A e$ into $f B f$ by $\theta(x)=V x V^{*}$. Put $\xi:=V^{*} \hat{f} \in L^{2}(e M f) \subset L^{2}(r M r)$. It holds that $x \xi=\xi \theta(x)$ for any $x \in e A e$. Take a polar decomposition of $\xi$ in $L^{2}(r M r)$ and denote its pole by $v \in r M r$. Then we have $v=e v=v f$ and $x v=v \theta(x)$ for any $x \in e A e$. This means $A \preceq_{r M r} q B q$.

We next consider the case that the subalgebra $B$ is of type III. In the case, equivalent conditions above no longer hold, but still there are some fundamental properties. For simplicity, from now on, we assume that subalgebras $A$ and $B$ are of type $\mathrm{II}_{1}$ or III. We write as $1_{A}$ and $1_{B}$ the units of $A$ and $B$.

Proposition 2.3.8. The following conditions are equivalent.

(i) We have $A \preceq_{M} B$.

(ii) There are a nonzero normal $*$-homomorphism $\pi: A \bar{\otimes} \mathbb{C} e_{1,1} \rightarrow B \bar{\otimes} \mathbb{M}_{n}$ for some $n \in \mathbb{N}$ and a nonzero partial isometry $w \in\left(1_{A} \otimes e_{1,1}\right)\left(M \bar{\otimes} \mathbb{M}_{n}\right)$ such that $w \pi(x)=\left(x \otimes e_{1,1}\right) w$ for all $x \in A$, where $\left(e_{i, j}\right)_{i, j}$ is a fixed matrix unit in $\mathbb{M}_{n}$.

Proof. The case that $B$ is of type $\mathrm{II}_{1}$ was already discussed in [25, Proposition 3.1]. So we assume that $B$ is of type III.

Suppose $A \preceq_{M} B$ and take $e, f, v$ and $\theta$ as in the definition. If $A$ is of type III, then $e \sim z_{A}(e)$ in $A$ with a partial isometry $u \in A$ such that $u u^{*}=e$ and $u^{*} u=z_{A}(e)$, where $z_{A}(e)$ is the central support projection of $e$ in $A$. The composite map

$$
A \stackrel{\times z_{A}(e)}{\longrightarrow} A z_{A}(e) \stackrel{\mathrm{Ad} u}{\longrightarrow} e A e \stackrel{\theta}{\longrightarrow} f B f
$$

and the partial isometry $u^{*} v$ satisfy condition (ii) (for $n=1$ ). If $A$ is of type $\mathrm{II}_{1}$, then the same argument as in [25, Proposition 3.1] works.

Suppose next condition (ii). The central support of $\pi\left(1_{A}\right)$ in $B \bar{\otimes} \mathbb{M}_{n}$ is of the form $z_{B} \otimes 1$ for some central projection $z_{B} \in B$. Then since central supports of $\pi\left(1_{A}\right)$ and $z_{B} \otimes e_{1,1}$ are same in the type III algebra $B \bar{\otimes} \mathbb{M}_{n}$, we have $\pi\left(1_{A}\right) \sim z_{B} \otimes e_{1,1}$ with a partial isometry $u \in B \bar{\otimes} \mathbb{M}_{n}$ such that $u^{*} u=\pi\left(1_{A}\right)$ and $u u^{*}=z_{B} \otimes e_{1,1}$. Then the composite map

$$
A \bar{\otimes} \mathbb{C} e_{1,1} \stackrel{\pi}{\longrightarrow} \pi\left(1_{A}\right)\left(B \bar{\otimes} \mathbb{M}_{n}\right) \pi\left(1_{A}\right) \stackrel{\operatorname{Ad} u}{\longrightarrow}\left(z_{B} \otimes e_{1,1}\right)\left(B \bar{\otimes} \mathbb{M}_{n}\right)\left(z_{B} \otimes e_{1,1}\right)=B z_{B} \bar{\otimes} \mathbb{C} e_{1,1}
$$

and the partial isometry $w u^{*} \in\left(1_{A} \otimes e_{1,1}\right)\left(M \bar{\otimes} \mathbb{M}_{n}\right)\left(z_{B} \otimes e_{1,1}\right) \subset M \bar{\otimes} \mathbb{C} e_{1,1}$ work by identifying $M \bar{\otimes} \mathbb{C} e_{1,1} \simeq M$.

From the characterization, it is easy to deduce the following properties. 
Corollary 2.3.9. The following statements are true.

(i) If $p A p \preceq_{M} B$ for some $p \in A$ or $A^{\prime} \cap M$, then $A \preceq_{M} B$.

(ii) If $A \preceq_{M} q B q$ for some $q \in B$ or $B^{\prime} \cap M$, then $A \preceq_{M} B$.

(iii) If $A \preceq_{M} B$, then $D \preceq_{M} B$ for any type $\mathrm{II}_{1}$ or III unital subalgebra $D \subset A$.

The next lemma is a special case of [26, Lemma 3.5], but it treats type III subalgebras.

Lemma 2.3.10. Assume that $M$ and $B$ are of the form $M=M_{1} \bar{\otimes} M_{2}$ and $B=M_{1}$ for von Neumann algebras $M_{1}$ and $M_{2}$. Assume that $M_{2}$ is of type III and $A^{\prime} \cap 1_{A} M 1_{A}$ is of type $\mathrm{II}_{1}$ or III. If $A \preceq_{M} M_{1}$, then $M_{2} \preceq_{M} A^{\prime} \cap 1_{A} M 1_{A}$.

Proof. Take $e \in A, f \in M_{1}, \theta$, and $v \in e M f$ as in the definition of $A \preceq_{M} M_{1}$. Write $p=v v^{*}$ and $q:=v^{*} v$. We have an inclusion $v^{*} p(e A e) p v \subset q \theta(e A e)$ as subalgebras of $q M q$. Taking relative commutants in $q M q$, we have

$$
q\left(\theta(e A e)^{\prime} \cap f M f\right) q=(q \theta(e A e))^{\prime} \cap q M q \subset\left(v^{*} e A e v\right)^{\prime} \cap q M q=v^{*} p\left((e A e)^{\prime} \cap e M e\right) p v .
$$

This implies $q\left(\theta(e A e)^{\prime} \cap f M f\right) q \preceq_{M} p\left((e A e)^{\prime} \cap e M e\right) p$ with the partial isometry $v$. Note that $\theta(e A e)^{\prime} \cap f M f$ is of the form $\left(\theta(e A e)^{\prime} \cap f M_{1} f\right) \bar{\otimes} M_{2}$ and hence is of type III. So by the previous lemma, we have $\theta(e A e)^{\prime} \cap f M f \preceq_{M}(e A e)^{\prime} \cap e M e=e\left(A^{\prime} \cap 1_{A} M 1_{A}\right) e$ and hence $f M_{2} \preceq_{M} e\left(A^{\prime} \cap 1_{A} M 1_{A}\right) e$, since $f M_{2} \subset \theta(e A e)^{\prime} \cap f M f$. Again by the lemma, we have $M_{2} \preceq_{M} A^{\prime} \cap 1_{A} M 1_{A}$.

\subsection{Approximately containment in continuous cores}

In the subsection, we introduce a notion of approximately containment formulated by Vaes [27, Section 2]. Our notion here is a slightly generalized but essentially the same one. Since we follow the same strategy as in [27, Section 2], we basically omit the proofs.

Let $\mathbb{G}$ be a compact quantum group and $h$ its Haar state. During the subsection, we consider following two cases at the same time.

- Case 1. Our target is the continuous core $L^{\infty}(\mathbb{G}) \rtimes_{\sigma^{h}} \mathbb{R}$ and its canonical trace, denoted by $M$ and Tr.

- Case 2. The quantum group $\mathbb{G}$ is of Kac type and the dual $\hat{\mathbb{G}}$ acts on a semifinite tracial von Neumann algebra $\left(N, \operatorname{Tr}_{N}\right)$ as a trace preserving action. Our target is the crossed product von Neumann algebra $\hat{\mathbb{G}} \ltimes N$ and its canonical trace, which is also denoted by $M$ and Tr.

For a discrete quantum subgroup $\hat{\mathbb{H}} \subset \hat{\mathbb{G}}$, we write $M_{\hat{\mathbb{H}}}:=L^{\infty}(\mathbb{H}) \rtimes_{\sigma^{h}} \mathbb{R}$ or $\hat{\mathbb{H}} \ltimes N$. For $\mathcal{E}, \mathcal{F} \subset \operatorname{Irred}(\mathbb{G})$ and $x, y \in \operatorname{Irred}(\mathbb{G})$, we use the notation

$$
\begin{aligned}
\mathcal{E F} & :=\{z \in \operatorname{Irred}(\mathbb{G}) \mid z \in e \otimes f \text { for some } e \in \mathcal{E}, f \in \mathcal{F}\}, \\
x \mathcal{E} y & :=\{z \in \operatorname{Irred}(\mathbb{G}) \mid z \in x \otimes e \otimes y \text { for some } e \in \mathcal{E}\} .
\end{aligned}
$$

Let $\mathcal{S}$ be a family of discrete quantum subgroups of $\hat{\mathbb{G}}$. We say a subset $\mathcal{F} \subset \operatorname{Irred}(\mathbb{G})$ is small relative to $\mathcal{S}$ if it is contained in a finite union of subsets of the form $x \operatorname{Irred}(\mathbb{H}) y$ for some $x, y \in \operatorname{Irred}(\mathbb{G})$ and $\hat{\mathbb{H}} \in \mathcal{S}$. For any subset $\mathcal{F} \subset \operatorname{Irred}(\mathbb{G})$, we write the orthogonal projection from $L^{2}(\mathbb{G}) \otimes L^{2}(\mathbb{R})$ onto $L^{2}(\mathcal{F}) \otimes L^{2}(\mathbb{R})\left(\right.$ or $L^{2}(\mathbb{G}) \otimes L^{2}(N)$ onto $L^{2}(\mathcal{F}) \otimes L^{2}(N)$ ) as $P_{\mathcal{F}}$, where $L^{2}(\mathcal{F})$ is the closed subspace spanned by all $u_{i, j}^{x}$ for $x \in \mathcal{F}$. For a subgroup $\hat{\mathbb{H}}$, the restriction of $P_{\operatorname{Irred}(\mathbb{H})}$ on $M$ is the trace preserving conditional expectation onto $M_{\hat{\mathbb{H}}}$. We write $\mathcal{N}_{\operatorname{Tr}}:=\left\{a \in M \mid \operatorname{Tr}\left(a^{*} a\right)<\infty\right\}$ and note that any element $a \in \mathcal{N}_{\operatorname{Tr}}$ has a Fourier expansion in $L^{2}(M, \operatorname{Tr})$ written as $a=\sum_{x \in \operatorname{Irred}(\mathbb{G}), i, j} u_{i, j}^{x} a_{i, j}^{x}$ for $a_{i, j}^{x}=E\left(u_{i, j}^{x *} a\right)$, where we take $\left(u_{i, j}^{x}\right)$ as an orthogonal family in $L^{2}(\mathbb{G})$ and $E$ is the Tr-preserving conditional expectation onto $L \mathbb{R}$ or $N$. 
Definition 2.4.1. Let $\mathcal{V}$ be a norm bounded subset in $\mathcal{N}_{\operatorname{Tr}} \subset M$. We say $\mathcal{V}$ is approximately contained in $M^{\mathcal{S}}$, and denote by $\mathcal{V} \subset$ approx $M^{\mathcal{S}}$, if for any $\epsilon>0$, there is $\mathcal{F} \subset \operatorname{Irred}(\mathbb{G})$ which is small relative to $\mathcal{S}$ such that

$$
\left\|b-P_{\mathcal{F}}(b)\right\|_{2}=\left\|P_{\mathcal{F} c}(b)\right\|_{2}<\epsilon
$$

for all $b \in \mathcal{V}$.

We also use the notation $B \subset_{\text {approx }} M^{\mathcal{S}}$ when $(B)_{1} \subset_{\text {approx }} M^{\mathcal{S}}$ for a subalgebra $B \subset M$, and $\mathcal{V} \subset$ approx $L^{\infty}(\mathbb{H}) \rtimes \mathbb{R}$ or $\hat{\mathbb{H}} \ltimes N$ when $\mathcal{S}=\{\hat{\mathbb{H}}\}$.

We start with a simple lemma.

Lemma 2.4.2. For any $\mathcal{F} \subset \operatorname{Irred}(\mathbb{G}), u^{a} \in \operatorname{span}\left\{u_{i, j}^{a} \mid i, j=1, \ldots, n_{a}\right\}$ for $a=x, y \in$ $\operatorname{Irred}(\mathbb{G})$, and $b \in \mathcal{N}_{\operatorname{Tr}}$, we have

$$
\left\|P_{\mathcal{F}}\left(u^{x *} b u^{y}\right)\right\|_{2} \leq\left\|u^{x *}\right\|\left\|P_{x \mathcal{F} \bar{y}}(b)\right\|_{2}\left\|u^{y}\right\| .
$$

Proof. We first assume $y=\epsilon$, the trivial corepresentation. Write $b=\sum_{z \in \operatorname{Irred}(\mathbb{G}), p, q} u_{p, q}^{z} b_{p, q}^{z}$ for some $b_{p, q}^{z} \in L \mathbb{R}($ or $N)$. For $z \in \operatorname{Irred}(\mathbb{G}),(\bar{x} \otimes z) \cap \mathcal{F} \neq \emptyset$ is equivalent to $z \in x \mathcal{F}$. Hence we have

$$
\begin{aligned}
\left\|P_{\mathcal{F}}\left(u^{x *} b\right)\right\|_{2} & =\left\|\sum_{z \in \operatorname{Irred}(\mathbb{G}), p, q} P_{\mathcal{F}}\left(u^{x *} u_{p, q}^{z} b_{p, q}^{z}\right)\right\|_{2} \\
& =\left\|\sum_{z \in x \mathcal{F}, p, q} P_{\mathcal{F}}\left(u^{x *} u_{p, q}^{z} b_{p, q}^{z}\right)\right\|_{2} \\
& \leq\left\|u^{x *}\right\|\left\|\sum_{z \in x \mathcal{F}, p, q} u_{p, q}^{z} b_{p, q}^{z}\right\|_{2}=\left\|u^{x *}\right\|\left\|P_{x \mathcal{F}}(b)\right\|_{2} .
\end{aligned}
$$

By the same manner, we also have $\left\|P_{\mathcal{F}}\left(b u^{y}\right)\right\|_{2} \leq\left\|P_{\mathcal{F} \bar{y}}(b)\right\|_{2}\left\|u^{y}\right\|$. Hence

$$
\left\|P_{\mathcal{F}}\left(u^{x *} b u^{y}\right)\right\|_{2} \leq\left\|u^{x *}\right\|\left\|P_{x \mathcal{F}}\left(b u^{y}\right)\right\|_{2} \leq\left\|u^{x *}\right\|\left\|P_{x \mathcal{F} \bar{y}}(b)\right\|_{2}\left\|u^{y}\right\| .
$$

By the lemma, we can prove the following lemma, which corresponds to [27, Lemma $2.3]$.

Lemma 2.4.3. Let $\mathcal{V} \subset \mathcal{N}_{\text {Tr }}$ be a norm bounded subset. If $\mathcal{V} \subset$ approx $M^{\mathcal{S}}$, then $x \mathcal{V} y \subset$ approx $M^{\mathcal{S}}$ for any $x, y \in \mathcal{N}_{\operatorname{Tr}}$.

Let $p \in M$ be a Tr-finite projection and $\left(v_{\lambda}\right)_{\lambda}$ be a bounded net in pMp. If $\left\|P_{\mathcal{F}}\left(v_{\lambda}\right)\right\|_{2} \rightarrow$ 0 for all $\mathcal{F}$ which is small relative to $\mathcal{S}$, then $\left\|P_{\operatorname{Irred}(\mathbb{H})}\left(b^{*} v_{\lambda} a\right)\right\|_{2} \rightarrow 0$ for all $a, b \in p M$ and all $\hat{\mathbb{H}} \in \mathcal{S}$.

To continue our argument, we need one more assumption which is an opposite phenomena to the second statement in the last lemma. More precisely we need the following condition:

- For any $\hat{\mathbb{H}} \in \mathcal{S}$, Tr-finite projection $p \in M$, and any net $\left(w_{\lambda}\right)_{\lambda}$ in $\mathcal{U}(p M p)$, if $\left\|P_{\text {Irred }(\mathbb{H})}\left(b^{*} w_{\lambda} a\right)\right\|_{2}$ converges to 0 for all $a, b \in p M$, then $\left\|P_{x \operatorname{Irred}(\mathbb{H}) \bar{y}}\left(w_{\lambda}\right)\right\|_{2}$ also converges to 0 for all $x, y \in \operatorname{Irred}(\mathbb{G})$.

When we treat a discrete group, since the inequality in Lemma 2.4.2 becomes the equality (because $\left.P_{\mathcal{F}}\left(\lambda_{x}^{*} b \lambda_{y}\right)=P_{x \mathcal{F} y^{-1}}(b)\right)$, this condition trivially holds. However, in the quantum situation, this equality is no longer true and hence we have to assume that our target $\hat{\mathbb{G}}$ and $\mathcal{S}$ satisfy this condition. Fortunately our main target, direct product quantum groups, always satisfy the condition. 
Lemma 2.4.4. Assume either that (i) $\hat{\mathbb{G}}$ is a group or (ii) $\hat{\mathbb{G}}$ is a direct product quantum group $\hat{\mathbb{G}}_{1} \times \cdots \times \hat{\mathbb{G}}_{m}$ and $\mathcal{S}$ consists of subgroups generated by some of $\hat{\mathbb{G}}_{i}$ for $i=1, \ldots, m$. Then the condition above holds.

Proof. The assumption (i) was already mentioned just before the lemma. So we assume (ii). Take any $\hat{\mathbb{H}} \in \mathcal{S}$ which is generated by some $\hat{\mathbb{G}}_{i}$. Exchanging their indices and writing $\hat{\mathbb{G}}=\hat{\mathbb{H}}_{1} \times \hat{\mathbb{H}}_{2}$, where $\hat{\mathbb{H}}_{1}:=\hat{\mathbb{G}}_{1} \times \cdots \times \hat{\mathbb{G}}_{n}$ and $\hat{\mathbb{H}}_{2}:=\hat{\mathbb{G}}_{n+1} \times \cdots \times \hat{\mathbb{G}}_{m}$ for some $n$, we may assume $\hat{\mathbb{H}}=\left\{\epsilon_{1}\right\} \times \hat{\mathbb{H}}_{2}$. Here $\epsilon_{1}$ is the unit of $\hat{\mathbb{H}}_{1}$. In the case, for any $x, y \in \operatorname{Irred}(\mathbb{G})$ there is a finite subset $\mathcal{F} \subset \operatorname{Irred}\left(\mathbb{H}_{1}\right)$ such that $x \operatorname{Irred}(\mathbb{H}) y \subset \mathcal{F} \times \operatorname{Irred}\left(\mathbb{H}_{2}\right)$. Since $P_{\mathcal{F} \times \operatorname{Irred}\left(\mathbb{H}_{2}\right)}=\sum_{z \in \mathcal{F}} P_{\{z\} \times \operatorname{Irred}\left(\mathbb{H}_{2}\right)}$, we may assume $x \in \operatorname{Irred}\left(\mathbb{H}_{1}\right)$ and $y$ is trivial.

Take a Fourier expansion of $w_{\lambda}$ along $\hat{\mathbb{H}}_{1}$, namely, decompose $w_{\lambda}=\sum_{z \in \operatorname{Irred}\left(\mathbb{H}_{1}\right), k, l} u_{k, l}^{z}\left(w_{\lambda}\right)_{k, l}^{z}$ for $\left(w_{\lambda}\right)_{k, l}^{z}=P_{\operatorname{Irred}\left(\mathbb{H}_{2}\right)}\left(u_{k, l}^{z *} w_{\lambda}\right) \in M_{\widehat{\mathbb{H}}_{2}}$, where $\left(u_{k, l}^{z}\right)_{k, l}^{z}$ is taken as an orthogonal system. Then for any $u_{i, j}^{x}$ we have

$$
0 \leftarrow\left\|u_{i, j}^{x}\right\|_{2}^{-2}\left\|P_{\operatorname{Irred}(\mathbb{H})}\left(u_{i, j}^{x *} w_{\lambda}\right)\right\|_{2}=\left\|u_{i, j}^{x}\right\|_{2}^{-2}\left\|\sum_{y, k, l} h\left(u_{i, j}^{x *} u_{k, l}^{y}\right)\left(w_{\lambda}\right)_{k, l}^{y}\right\|_{2}=\left\|\left(w_{\lambda}\right)_{i, j}^{x}\right\|_{2},
$$

and hence

$$
\left\|P_{\{x\} \times \operatorname{Irred}\left(\mathbb{H}_{2}\right)}\left(\omega_{\lambda}\right)\right\|_{2}=\left\|\sum_{i, j} u_{i, j}^{x}\left(w_{\lambda}\right)_{i, j}^{x}\right\|_{2} \leq \sum_{i, j}\left\|\left(w_{\lambda}\right)_{i, j}^{x}\right\|_{2} \rightarrow 0 .
$$

From now on, we assume that $\mathbb{G}$ and $\mathcal{S}$ satisfy the condition above. Then we can follow all the proofs in [27, Section 2] and get the following four statements.

Lemma 2.4.5. Let $p$ be a Tr-finite projection in $M$ and $B \subset p M p$ be a von Neumann subalgebra generated by a group of unitaries $\mathcal{G} \subset \mathcal{U}(B)$. The following statements are equivalent.

- For every $\hat{\mathbb{H}} \in \mathcal{S}$, we have $B \npreceq{ }_{M} M_{\hat{\mathbb{H}}}$.

- There exists a net of unitaries $\left(w_{i}\right)$ in $\mathcal{G}$ such that $\left\|P_{\mathcal{F}}\left(w_{i}\right)\right\|_{2} \rightarrow 0$ for every subset $\mathcal{F} \subset \operatorname{Irred}(\mathbb{G})$ which is small relative to $\mathcal{S}$.

Lemma 2.4.6. Let $p$ be a Tr-finite projection in $M$ and $B \subset p M p$ be a von Neumann subalgebra. The following statements are equivalent.

1. There exists an $\hat{\mathbb{H}} \in \mathcal{S}$ such that $B \preceq_{M} M_{\hat{\mathbb{H}}}$.

2. There exists a nonzero projection $q \in B^{\prime} \cap p M p$ such that $(B q)_{1} \subset$ approx $M^{\mathcal{S}}$.

Also the following two statements are equivalent.

a. For every nonzero projection $q \in B^{\prime} \cap p M p$, there exists an $\hat{\mathbb{H}} \in \mathcal{S}$ such that $B q \preceq_{M}$ $M_{\hat{\mathbb{H}}}$.

b. We have $(B)_{1} \subset_{\text {approx }} M^{\mathcal{S}}$.

Proposition 2.4.7. The set of projections

$$
\mathcal{P}:=\left\{q_{0} \in B^{\prime} \cap p M p \mid\left(B q_{0}\right)_{1} \subset_{\text {approx }} M^{\mathcal{S}}\right\}
$$

attains its maximum in a unique projection $q \in \mathcal{P}$. This projection belongs to $\mathcal{Z}\left(\mathcal{N}_{p M p}(B)^{\prime \prime}\right)$. 
Lemma 2.4.8. Let $\mathcal{S}_{1}, \mathcal{S}_{2}$ and $\mathcal{S}$ be families of discrete quantum subgroups of $\hat{\mathbb{G}}$. Assume that for any $\mathcal{F}_{i}$ which is small relative to $\mathcal{S}_{i}, \mathcal{F}_{1} \cap \mathcal{F}_{2}$ is small relative to $\mathcal{S}$. If $(B)_{1} \subset$ approx $M^{\mathcal{S}_{i}}$ for $i=1,2$, then $(B)_{1} \subset_{\text {approx }} M^{\mathcal{S}}$.

Remark 2.4.9. As mentioned in [27, Lemma 2.7], when $\hat{\mathbb{G}}$ is a discrete group $\Gamma$, we can put $\mathcal{S}:=\left\{\Sigma_{1} \cap g \Sigma_{2} g^{-1} \mid \Sigma_{i} \in \mathcal{S}_{i}\right.$ for $i=1,2$ and $\left.g \in \Gamma\right\}$. For our main target, direct product quantum groups, we can put $\mathcal{S}:=\left\{\hat{\mathbb{H}}_{1} \cap \hat{\mathbb{H}}_{2} \mid \hat{\mathbb{H}}_{i} \in \mathcal{S}_{i}\right.$ for $\left.i=1,2\right\}$.

In the proof of main theorems, we often use the following lemma, which is an easy consequence of lemmas above.

Lemma 2.4.10. Let $p$ be a Tr-finite projection in $M$ and $B \subset p M p$ be a von Neumann subalgebra. Assume that $B^{\prime} \cap p M p$ is a factor. Then for any $\mathcal{S},(B)_{1} \subset$ approx $M^{\mathcal{S}}$ if and only if there exists an $\hat{\mathbb{H}} \in \mathcal{S}$ such that $B \preceq_{M} M_{\hat{\mathbb{H}}}$.

Proof. The only if direction is trivial. Assume $B \preceq_{M} M_{\hat{\mathbb{H}}}$ for some $\hat{\mathbb{H}} \in \mathcal{S}$. Then by Lemma 2.4.6, there is a nonzero projection $q_{0} \in B^{\prime} \cap p M p$ such that $\left(q_{0} B\right)_{1} \subset_{\text {approx }} M^{\mathcal{S}}$. Take the maximum projection $q \in B^{\prime} \cap p M p$ in Proposition 2.4.7 such that $(q B)_{1} \subset_{\text {approx }}$ $M^{\mathcal{S}}$. Since $q$ is non-zero and is contained in $B^{\prime} \cap p M p \cap \mathcal{Z}\left(\mathcal{N}_{p M p}(B)^{\prime \prime}\right) \subset \mathcal{Z}\left(B^{\prime} \cap p M p\right)=\mathbb{C}$, $q=1$ and we get $(B)_{1} \subset_{\text {approx }} M^{\mathcal{S}}$.

\section{Prime factorization results for type III factors}

In the section, we prove the main theorem. As we mentioned in Introduction, we begin our work by generalizing condition $(\mathrm{AO})$ to the tensor product setting. We then study locations of subalgebras and intertwiners inside cores of tensor products by Popa's intertwining method. We obtain factorization results for cores first, and then deduce factorization results for the original algebras.

\subsection{Condition ( $\mathrm{AO})$ for tensor product algebras}

As we mentioned, a discrete quantum group in $\mathcal{C}$ is bi-exact, and hence the associated von Neumann algebra satisfies condition (AO). In the subsection, we study some appropriate conditions on direct products of quantum groups in $\mathcal{C}$. Such an observation was first given in [15] for direct products and then generalized in [2, Section 15] with the notion of relative bi-exactness. Our approach here is close to the first one. In the subsection, we use the notation

$$
\mathbb{B}_{\mathbb{G}}:=\mathbb{B}\left(L^{2}(\mathbb{G})\right), \mathbb{K}_{\mathbb{G}}:=\mathbb{K}\left(L^{2}(\mathbb{G})\right), \quad \mathbb{B}_{\mathbb{R}}:=\mathbb{B}\left(L^{2}(\mathbb{R})\right), \mathbb{K}_{\mathbb{R}}:=\mathbb{K}\left(L^{2}(\mathbb{R})\right),
$$

for any compact quantum group $\mathbb{G}$ and the real numbers $\mathbb{R}$.

Let $\hat{\mathbb{G}}_{i}(i=1, \ldots, m)$ be discrete quantum groups in $\mathcal{C}$. By definition there are $\hat{\mathbb{H}}_{i}$ containing $\hat{\mathbb{G}}_{i}$ with nuclear $C^{*}$-algebras $\mathcal{C}_{l}^{i}$. For simplicity, we first assume $\hat{\mathbb{G}}_{i}=\hat{\mathbb{H}}_{i}$. We will get the same conclusions in the general case (Lemma 3.1.5). We use the notation

$$
\begin{aligned}
& \mathcal{C}_{l}:=\mathcal{C}_{l}^{1} \otimes_{\min } \cdots \otimes_{\min } \mathcal{C}_{l}^{m}, \quad \hat{\mathbb{G}}:=\hat{\mathbb{G}}_{1} \times \cdots \times \hat{\mathbb{G}}_{m}, \\
& \hat{\mathbb{G}}_{i}^{\prime}:=\hat{\mathbb{G}}_{1} \times \cdots \times \hat{\mathbb{G}}_{i-1} \times\left\{\epsilon_{i}\right\} \times \hat{\mathbb{G}}_{i+1} \times \cdots \times \hat{\mathbb{G}}_{m},
\end{aligned}
$$

where $\epsilon_{i}$ is the unit of $\hat{\mathbb{G}}_{i}$. Note that $\mathcal{C}_{l}$ is also nuclear and contains $C_{\text {red }}(\mathbb{G})$. Consider a multiplication map

$$
\nu: \mathcal{C}_{l} \otimes_{\text {alg }} C_{\text {red }}(\mathbb{G})^{\text {op }} \ni a \otimes b^{\text {op }} \mapsto a b^{\text {op }} \in \mathbb{B}\left(L^{2}(\mathbb{G})\right),
$$


This is a well defined linear map but not a $*$-homomorphism in general. In fact, $\nu$ is a $*$-homomorphism if and only if commutators $\left[\nu\left(\mathcal{C}_{l}\right), \nu\left(C_{\text {red }}(\mathbb{G})^{\text {op }}\right)\right]$ are zero. If there is a non-unital $C^{*}$-algebra $J \subset \mathbb{B}\left(L^{2}(\mathbb{G})\right)$ which contains $\left[\nu\left(\mathcal{C}_{l}\right), \nu\left(C_{\text {red }}(\mathbb{G})^{\text {op }}\right)\right]$ and whose multiplier algebra $M(J)$ contains $\operatorname{ran} \nu$, then exchanging the range of $\nu$ from $\mathbb{B}\left(L^{2}(\mathbb{G})\right)$ with $M(J) / J, \nu$ becomes a $*$-homomorphism. In our situation, we can find an appropriate $J$ as follows.

Lemma 3.1.1. Denote

$$
\mathcal{K}_{i}:=\mathbb{B}_{\mathbb{G}_{1}} \otimes_{\min } \cdots \otimes_{\min } \mathbb{B}_{\mathbb{G}_{i-1}} \otimes_{\min } \mathbb{K}_{\mathbb{G}_{i}} \otimes_{\min } \mathbb{B}_{\mathbb{G}_{i+1}} \otimes_{\min } \cdots \otimes_{\min } \mathbb{B}_{\mathbb{G}_{n}} .
$$

Put $J:=\sum_{i} \mathcal{K}_{i}$. Then $J$ is a $C^{*}$-algebra containing $\left[\nu\left(\mathcal{C}_{l}\right), \nu\left(C_{\mathrm{red}}(\mathbb{G})^{\mathrm{op}}\right)\right]$ and the multiplier algebra $M(J)$ contains $\operatorname{ran} \nu$.

Proof. Since each $\mathcal{K}_{i}$ is an ideal in $\mathbb{B}_{\mathbb{G}_{1}} \otimes_{\min } \cdots \otimes_{\min } \mathbb{B}_{\mathbb{G}_{n}}, J$ is a $C^{*}$-algebra. Since ran $\nu$ is contained in $\mathbb{B}_{\mathbb{G}_{1}} \otimes_{\min } \cdots \otimes_{\min } \mathbb{B}_{\mathbb{G}_{n}}$, it is contained in $M(J)$. To show $\left[\nu\left(\mathcal{C}_{l}\right), \nu\left(C_{\text {red }}(\mathbb{G})^{\text {op }}\right)\right] \subset$ $J$, we have only to check $\left[\nu\left(\mathcal{C}_{l}^{i}\right), \nu\left(C_{\text {red }}\left(\mathbb{G}_{j}\right)^{\text {op }}\right)\right] \subset J$ for any $i, j$, because $J$ is an ideal in $M(J)$. The commutators are zero when $i \neq j$, and are contained in $\mathcal{K}_{i}$ when $i=j$.

We keep $J$ in the lemma. Then we get a $*$-homomorphism $\nu$ from $\mathcal{C}_{l} \otimes_{\text {alg }} C_{\text {red }}(\mathbb{G})^{\text {op }}$ into $M(J) / J$, which is bounded with respect to the max tensor product norm. Since $\mathcal{C}_{l}$ is nuclear, the max norm coincides with the minimal tensor norm. Finally restricting the map on $C_{\text {red }}(\mathbb{G}) \otimes_{\text {alg }} C_{\text {red }}(\mathbb{G})^{\text {op }}$, we get the following proposition which is an analogue of condition $(\mathrm{AO})$ on tensor product algebras.

Proposition 3.1.2. The $C^{*}$-algebra $C_{\mathrm{red}}(\mathbb{G})$ is exact and the multiplication map

$$
C_{\text {red }}(\mathbb{G}) \otimes_{\text {alg }} C_{\text {red }}(\mathbb{G})^{\mathrm{op}} \ni a \otimes b^{\mathrm{op}} \mapsto a b^{\mathrm{op}} \in M(J) / J
$$

is bounded with respect to the minimal tensor norm.

We next investigate a similar property on continuous cores. Recall that commutants of continuous cores are of the form

$$
\begin{aligned}
& L^{\infty}(\mathbb{G}) \rtimes \mathbb{R}=W^{*}\left\{\pi\left(L^{\infty}(\mathbb{G})\right), 1 \otimes \lambda_{t}(t \in \mathbb{R})\right\}, \\
& \left(L^{\infty}(\mathbb{G}) \rtimes \mathbb{R}\right)^{\prime}=\left(L^{\infty}(\mathbb{G}) \rtimes \mathbb{R}\right)^{\mathrm{op}}=W^{*}\left\{L^{\infty}(\mathbb{G})^{\mathrm{op}} \bar{\otimes} 1, \Delta^{i t} \otimes \rho_{t}(t \in \mathbb{R})\right\} .
\end{aligned}
$$

where $\pi$ is the canonical $*$-homomorphism into $\mathbb{B}\left(L^{2}(\mathbb{G}) \otimes L^{2}(\mathbb{R})\right), \Delta$ is the modular operator of the Haar state of $\mathbb{G}$, and $\rho_{t}$ is the right regular representation of $\mathbb{R}$. We go along a similar line to above by exchanging $C_{\text {red }}(\mathbb{G})$ with $C_{\text {red }}(\mathbb{G}) \rtimes_{\mathrm{r}} \mathbb{R}$, the reduced norm continuous crossed product, which is a dense subalgebra in the core.

Keep the notation above and let us first consider a multiplication map

$$
\mu: \mathcal{C}_{l} \otimes_{\mathrm{alg}} C_{\mathrm{red}}(\mathbb{G})^{\mathrm{op}} \ni a \otimes b \mapsto \pi(a)(b \otimes 1) \in \mathbb{B}\left(L^{2}(\mathbb{G}) \otimes L^{2}(\mathbb{R})\right) .
$$

In (the proof of) [10, Proposition 3.2.3], we verified that $M\left(\mathbb{K}_{\mathbb{G}} \otimes_{\min } \mathbb{B}_{\mathbb{R}}\right)$ contains the $C^{*}$-algebra $D$ which is generated by

- the image of $\mu$;

- $1 \otimes \lambda_{t}, \Delta^{i t} \otimes \rho_{t}(t \in \mathbb{R})$;

- $\int_{\mathbb{R}} f(s)\left(1 \otimes \lambda_{s}\right) \cdot d s, \int_{\mathbb{R}} f(s)\left(\Delta^{i s} \otimes \rho_{s}\right) \cdot d s\left(f \in L^{1}(\mathbb{R})\right)$

(for the fact, we do not need any assumption on $\mathbb{G})$. In our situation, since $\left[\mu\left(\mathcal{C}_{l}\right), \mu\left(C_{\text {red }}(\mathbb{G})^{\text {op }}\right)\right]$ is not contained in $\mathbb{K}_{\mathbb{G}} \otimes_{\min } \mathbb{B}_{\mathbb{R}}$, the algebra $M\left(\mathbb{K}_{\mathbb{G}} \otimes_{\min } \mathbb{B}_{\mathbb{R}}\right)$ should be exchanged by $M(\tilde{J})$ for some appropriate $\tilde{J}$. 
Lemma 3.1.3. Put $\tilde{J}:=J \otimes_{\min } \mathbb{B}_{\mathbb{R}}=\sum_{i} \mathcal{K}_{i} \otimes_{\min } \mathbb{B}_{\mathbb{R}}$. Then $\tilde{J}$ is a $C^{*}$-algebra containing $\left[\mu\left(\mathcal{C}_{l}\right), \mu\left(C_{\text {red }}(\mathbb{G})^{\text {op }}\right)\right] \subset \tilde{J}$ and the multiplier algebra $M(\tilde{J})$ contains $D$.

Proof. Since each $\mathcal{K}_{i} \otimes_{\text {min }} \mathbb{B}_{\mathbb{R}}$ is an ideal in $\mathbb{B}_{\mathbb{G}_{1}} \otimes_{\min } \cdots \otimes_{\min } \mathbb{B}_{\mathbb{G}_{n}} \otimes_{\min } \mathbb{B}_{\mathbb{R}}$, $\tilde{J}$ is a $C^{*}$ algebra. Obviously $1 \otimes \lambda_{t}$ and $\Delta^{i t} \otimes \rho_{t}(t \in \mathbb{R})$, and $\mu\left(C_{\text {red }}(\mathbb{G})^{\text {op }}\right)$ are contained in $\mathbb{B}_{\mathbb{G}_{1}} \otimes_{\min } \cdots \otimes_{\min } \mathbb{B}_{\mathbb{G}_{n}} \otimes_{\min } \mathbb{B}_{\mathbb{R}}$ and hence in $M(\tilde{J})$. Let $\left(p_{j}^{i}\right)_{j}$ be an increasing net of central projections in $\hat{\lambda}\left(c_{0}\left(\hat{\mathbb{G}}_{i}\right)\right) \subset \mathbb{K}_{\mathbb{G}_{i}}$ converging to 1 strongly, which automatically commute with $\Delta^{i t}(t \in \mathbb{R})$ and $\hat{\lambda}\left(\ell^{\infty}\left(\hat{\mathbb{G}}_{i}\right)\right)$. Then $\int_{\mathbb{R}} f(s)\left(1 \otimes \lambda_{s}\right) \cdot d s$ and $\int_{\mathbb{R}} f(s)\left(\Delta^{i s} \otimes \rho_{s}\right) \cdot d s$ $\left(f \in L^{1}(\mathbb{R})\right)$, and $\mu\left(\ell^{\infty}(\hat{\mathbb{G}})\right)$ commute with $1_{\mathbb{G}_{i}^{\prime}} p_{j}^{i} \otimes 1_{\mathbb{R}} \in \mathcal{K}_{i} \otimes_{\min } \mathbb{B}_{\mathbb{R}}$. So they are contained in $M\left(\mathcal{K}_{i} \otimes_{\min } \mathbb{B}_{\mathbb{R}}\right)$ for all $i$ and hence in $M(\tilde{J})$. Let $\left(u_{k, l}^{x}\right)$ be a basis of the dense Hopf *-algebra of $C_{\text {red }}(\mathbb{G})$, which is orthogonal in $L^{2}(\mathbb{G})$. Then since $\sigma_{t}^{h}\left(u_{k, l}^{x}\right)=\left(\lambda_{k, l}^{x}\right)^{i t} u_{k, l}^{x}$ $(t \in \mathbb{R})$ for some $\lambda_{k, l}^{x}>0, \pi\left(u_{k, l}^{x}\right)$ is of the form $u_{k, l}^{x} \otimes f_{k, l}^{x}$, where $f_{k, l}^{x} \in L^{\infty}(\mathbb{R})$ is given by $f_{k, l}^{x}(t)=\left(\lambda_{k, l}^{x}\right)^{-i t}$. This is contained in $M(\tilde{J})$ and hence $\mu\left(\mathcal{C}_{l}\right)$ is contained in $M(\tilde{J})$.

To prove $\left[\mu\left(\mathcal{C}_{l}\right), \mu\left(C_{\text {red }}(\mathbb{G})^{\text {op }}\right)\right] \subset \tilde{J}$, it suffice to show $\left[\mu\left(\mathcal{C}_{l}^{i}\right), \mu\left(C_{\text {red }}\left(\mathbb{G}_{j}\right)^{\text {op }}\right)\right] \subset \tilde{J}$ for any $i, j$. The commutators are zero when $i \neq j$, and are contained in $\mathcal{K}_{i} \otimes_{\text {min }} \mathbb{B}_{\mathbb{R}}$ when $i=j$.

We keep $\tilde{J}$ in the lemma. Exchange the range of $\mu$ with $M(\tilde{J}) / \tilde{J}$ and get a $*-$ homomorphism. It is bounded with respect to the minimal tensor norm since $\mathcal{C}_{l}$ is nuclear. Then consider $(\mathbb{R} \times \mathbb{R})$-actions on $\mathcal{C}_{l} \otimes_{\min } C_{\text {red }}(\mathbb{G})^{\text {op }}$ and $M(\tilde{J}) / \tilde{J}$ given by $(s, t) \mapsto \operatorname{Ad}\left(\Delta^{i s} \otimes \Delta^{i t}\right)$ and $(s, t) \mapsto \operatorname{Ad}\left(\left[1 \otimes \lambda_{s}\right]\left[\Delta^{i t} \otimes \rho_{t}\right]\right)$ respectively. It is easy to verify that $\mu$ is $(\mathbb{R} \times \mathbb{R})$-equivariant. Since $M(\tilde{J})$ contains $D$ and $\mathbb{R} \times \mathbb{R}$ is amenable, we get the following map

$$
\left(\mathcal{C}_{l} \rtimes_{\mathrm{r}} \mathbb{R}\right) \otimes_{\min }\left(C_{\mathrm{red}}(\mathbb{G}) \rtimes_{\mathrm{r}} \mathbb{R}\right)^{\mathrm{op}} \simeq\left(\mathcal{C}_{l} \otimes_{\min } C_{\mathrm{red}}(\mathbb{G})^{\mathrm{op}}\right) \rtimes_{\mathrm{r}}(\mathbb{R} \times \mathbb{R}) \rightarrow M(\tilde{J}) / \tilde{J} .
$$

Restricting the map, we get the following proposition, which is also an analogue of condition $(\mathrm{AO})$ on tensor products.

Proposition 3.1.4. The $C^{*}$-algebra $C_{\mathrm{red}}(\mathbb{G}) \rtimes_{\mathrm{r}} \mathbb{R}$ is exact and the multiplication map

$$
\left(C_{\text {red }}(\mathbb{G}) \rtimes_{\mathrm{r}} \mathbb{R}\right) \otimes_{\text {alg }}\left(C_{\text {red }}(\mathbb{G}) \rtimes_{\mathrm{r}} \mathbb{R}\right)^{\mathrm{op}} \ni a \otimes b^{\mathrm{op}} \mapsto a b^{\mathrm{op}} \in M(\tilde{J}) / \tilde{J}
$$

is bounded with respect to the minimal tensor norm.

Recall that we assumed $\hat{\mathbb{G}}_{i}=\hat{\mathbb{H}}_{i}$ at the first stage in the subsection. In the general case, cutting by $e_{\mathbb{G}_{i}}$, which is the projection from $L^{2}\left(\mathbb{H}_{i}\right)$ onto $L^{2}\left(\mathbb{G}_{i}\right)$, we can easily deduce the following lemma (e.g. [12, Lemma 3.3.1]).

Lemma 3.1.5. Propositions 3.1.2 and 3.1 .4 are true for general $\hat{\mathbb{G}}_{i}$ in $\mathcal{C}$.

Remark 3.1.6. In the end of Subsection 2.2, we observed that the Haar state preserving conditional expectation $E_{h}$ onto $L^{\infty}(\mathbb{G})_{h}$ gives a conditional expectation from $C_{\text {red }}(\mathbb{G})$ onto $C_{\text {red }}(\mathbb{G})_{h}$. By the same argument above, we can also exchange all objects of $\hat{\mathbb{G}}_{i}$ in Proposition 3.1 .2 with $C_{\text {red }}\left(\mathbb{G}_{i}\right)_{h}, L^{\infty}\left(\mathbb{G}_{i}\right)_{h}$ and $L^{2}\left(L^{\infty}\left(\mathbb{G}_{i}\right)_{h}\right)$. Hence by Ozawa-Popa's method, we can prove prime factorization results for $L^{\infty}\left(\mathbb{G}_{1}\right)_{h_{1}} \bar{\otimes} \cdots \bar{\otimes} L^{\infty}\left(\mathbb{G}_{n}\right)_{h_{n}}$ if each tensor component is a non-amenable $\mathrm{II}_{1}$ factor.

\subsection{Location of subalgebras}

In this subsection, we give a key observation for our factorization results. Our condition (AO) phenomena is used only to prove the following proposition. It is a generalization of [10, Theorem C] and its origin is [13, Theorem 4.6] (see also [11, Theorem 5.3.3]). 
Let $\hat{\mathbb{G}}_{i}(i=1, \ldots, m)$ be discrete quantum groups in $\mathcal{C}$ and $h_{i}$ be Haar states of $\mathbb{G}_{i}$. Write $h:=h_{1} \otimes \cdots \otimes h_{n}$. We use the notation in the previous section such as $\hat{\mathbb{G}}$, $\hat{\mathbb{G}}_{i}^{\prime}$ and $\tilde{J}$.

Proposition 3.2.1. Let $p$ be a Tr-finite projection in $C_{h}\left(L^{\infty}(\mathbb{G})\right)\left(=: C_{h}\right)$, where $\operatorname{Tr}$ is the canonical trace on $C_{h}$, and $N \subset p C_{h} p$ a von Neumann subalgebra. Then we have either one of the following statements:

(i) The relative commutant $N^{\prime} \cap p C_{h} p$ is amenable.

(ii) We have $N \preceq_{C_{h}} L^{\infty}\left(\mathbb{G}_{i}^{\prime}\right) \rtimes \mathbb{R}$ for some $i$.

Proof. We give only a sketch of the proof. By [11, Proposition 5.2.4], we may assume that $N$ is amenable. Suppose by contradiction that $N \swarrow_{r C_{h} r} q\left(L^{\infty}\left(\mathbb{G}_{i}^{\prime}\right) \rtimes \mathbb{R}\right) q$ for any $i$ and any Tr-finite projection $q \in L^{\infty}\left(\mathbb{G}_{i}^{\prime}\right) \rtimes \mathbb{R}$ and $r:=p \vee q$.

Define a proper conditional expectation $\Psi_{N}: \mathbb{B}\left(L^{2}\left(C_{h}\right)\right) \rightarrow N^{\prime}$. For any $i$, we will show $\Psi_{N}\left(e_{\mathbb{G}_{i}} \otimes 1_{\mathbb{G}_{i}^{\prime}} \otimes 1_{\mathbb{R}}\right)=0$, where $e_{\mathbb{G}_{i}}$ is the projection from $L^{2}\left(\mathbb{G}_{i}\right)$ onto $\mathbb{C} \hat{1}_{\mathbb{G}_{i}}$. This implies $\mathbb{K}\left(L^{2}\left(\mathbb{G}_{i}\right)\right) \otimes_{\min } \mathbb{B}\left(L^{2}\left(\mathbb{G}_{i}^{\prime}\right) \otimes L^{2}(\mathbb{R})\right) \subset \operatorname{ker} \Psi_{N}$ and hence $\tilde{J} \subset \operatorname{ker} \Psi_{N}$.

Let $q \in L^{\infty}\left(\mathbb{G}_{i}^{\prime}\right) \rtimes \mathbb{R}$ be any projection with $\operatorname{Tr}(q)<\infty$ and $z_{q}$ be the central support of $q$ in $L^{\infty}\left(\mathbb{G}_{i}^{\prime}\right) \rtimes \mathbb{R}$. Write $s:=z_{q}^{\mathrm{op}} \Psi_{N}\left(e_{\mathbb{G}_{i}} \otimes 1_{\mathbb{G}_{i}^{\prime}} \otimes 1_{\mathbb{R}}\right) z_{q}^{\mathrm{op}}$. Then $s$ is contained in $N^{\prime} \cap\left(\left(L^{\infty}\left(\mathbb{G}_{i}^{\prime}\right) \rtimes \mathbb{R}\right)^{\mathrm{op}}\right)^{\prime}$ and satisfies $s=s z_{q}^{\mathrm{op}} p$. Since $N \swarrow_{r C_{h} r} q\left(L^{\infty}\left(\mathbb{G}_{i}^{\prime}\right) \rtimes \mathbb{R}\right) q$, by Proposition 2.3.5. $\operatorname{Tr}_{i}(s)$ is zero or infinite, where $\operatorname{Tr}_{i}$ is the canonical trace on the basic construction of $L^{\infty}\left(\mathbb{G}_{i}^{\prime}\right) \rtimes \mathbb{R} \subset C_{h}$. This actually has a finite value because

$$
\begin{aligned}
\operatorname{Tr}_{i}(s) & \leq \operatorname{Tr}_{i}\left(\Psi_{N}\left(e_{\mathbb{G}_{i}} \otimes 1_{\mathbb{G}_{i}^{\prime}} \otimes 1_{\mathbb{R}}\right)\right) \\
& \leq \operatorname{Tr}_{i}\left(p\left(e_{\mathbb{G}_{i}} \otimes 1_{\mathbb{G}_{i}^{\prime}} \otimes 1_{\mathbb{R}}\right) p\right) \\
& \leq \operatorname{Tr}_{i}\left(\left(e_{\mathbb{G}_{i}} \otimes 1_{\mathbb{G}_{i}^{\prime}} \otimes 1_{\mathbb{R}}\right) E_{i}(p)\right) \\
& =\operatorname{Tr}\left(E_{i}(p)\right)=\operatorname{Tr}(p)<\infty,
\end{aligned}
$$

where $E_{i}$ is the Tr-preserving conditional expectation from $C_{h}$ onto $L^{\infty}\left(\mathbb{G}_{i}^{\prime}\right) \rtimes \mathbb{R}$. Thus we get $\operatorname{Tr}_{i}(s)=0$ and this means $\Psi_{N}\left(e_{\mathbb{G}_{i}} \otimes 1_{\mathbb{G}_{i}^{\prime}} \otimes 1_{\mathbb{R}}\right)=0$.

Now considering the composite map

$$
\left(C_{\mathrm{red}}(\mathbb{G}) \rtimes_{\mathrm{r}} \mathbb{R}\right) \otimes_{\min }\left(C_{\mathrm{red}}(\mathbb{G}) \rtimes_{\mathrm{r}} \mathbb{R}\right)^{\mathrm{op}} \stackrel{\mu}{\rightarrow} M(\tilde{J}) / \tilde{J} \stackrel{\Psi}{\rightarrow} N^{\prime},
$$

where $\mu$ is as in Proposition 3.1.4, we can follow the same method as in [13, Theorem 4.6] (or [11, Theorem 5.3.3]).

\subsection{Intertwiners inside type III algebras and continuous cores}

In the work of Ozawa and Popa on prime factorizations, they first found an intertwiner between tensor components of both sides and then constructed a unitary element which moves tensor components from one side to the other. In the subsection we study corresponding statements for type III factors. We recall the statement on $\mathrm{II}_{1}$ factors and then prove a similar one on type III factors.

Lemma 3.3.1 ([15, Proposition 12]). Let $M_{i}$ and $N_{i}$ be $\mathrm{II}_{1}$ factors with $M_{1} \bar{\otimes} M_{2}=N_{1} \bar{\otimes} N_{2}$ $(=: M)$. Assume $N_{1} \preceq_{M} M_{1}$. Then there is a unitary element $u \in M$ and a decomposition $M \simeq M_{1}^{t} \bar{\otimes} M_{2}^{1 / t}$ for some $t>0$ such that $u N_{1} u^{*} \subset M_{1}^{t}$.

Lemma 3.3.2. Let $M_{i}$ and $N_{i}$ be factors with $M_{1} \bar{\otimes} M_{2}=N_{1} \bar{\otimes} N_{2}(=: M)$. Assume that $M_{2}$ and $N_{2}$ are type III factors and $N_{1} \preceq_{M} M_{1}$. Then there is a partial isometry $u \in M$ such that $u^{*} u \in N_{1}, u u^{*} \in M_{1}$, and $u N_{1} u^{*} \subset u u^{*} M_{1} u u^{*}$. If moreover $M_{1}$ and $N_{1}$ are also type III factors, the element $u$ can be taken as a unitary element. 
Proof. By assumption, there are $e \in N_{1}, f \in M_{1}, v \in M$, and $\theta: e N_{1} e \rightarrow f M_{1} f$. Since $v v^{*} \in N_{1}^{\prime} \cap e M e=e N_{2}$ and $e N_{2}$ is a type III factor, we have $v v^{*} \sim e$ with a partial isometry $u \in e N_{2}$ such that $u u^{*}=e$ and $u^{*} u=v v^{*}$. Replacing $v$ with $u^{*} v$, we may assume that $e=v v^{*}$. Since $v^{*} v \in \theta(e A e)^{\prime} \cap f M f=\left(\theta(e A e)^{\prime} \cap f M_{1} f\right) \bar{\otimes} M_{2}$ and $\left(\theta(e A e)^{\prime} \cap f M_{1} f\right) \bar{\otimes} M_{2}$ is of type III, $v^{*} v \sim z \otimes 1_{M_{2}}$ for some $z \in \mathcal{Z}\left(\theta(e A e)^{\prime} \cap f M_{1} f\right)$ with a partial isometry $u \in\left(\theta(e A e)^{\prime} \cap f M_{1} f\right) \bar{\otimes} M_{2}$ such that $u u^{*}=z \otimes 1_{M_{2}}$ and $u^{*} u=v^{*} v$. Replacing $\theta$ and $v$ with $\left(z \otimes 1_{M_{2}}\right) \theta(x)$ for $x \in e N_{1} e$ and $v u^{*}$, we may assume $f=v^{*} v$. So the first statement holds.

We next assume $M_{1}$ and $N_{1}$ are of type III. Then since $e \sim 1_{N_{1}}$ in $N_{1}$ and $f \sim 1_{M_{1}}$ in $M_{1}$, replacing $v$, we can assume $v v^{*}=v^{*} v=1$.

\subsection{Proof of Theorem A}

We start with two simple lemmas.

Lemma 3.4.1. Let $M$ be a von Neumann algebra and $N \subset M$ a subfactor with a faithful normal conditional expectation $E_{N}$. Let $\phi$ be a faithful normal state on $M$ with $\phi \circ E_{N}=\phi$. If $M=N \vee\left(N^{\prime} \cap M\right)$, then we have an isomorphism $N \bar{\otimes}\left(N^{\prime} \cap M\right) \ni a \otimes b \mapsto a b \in M$.

Proof. See the proof of [24, Lemma XIV.2.5].

Lemma 3.4.2. Let $M_{i}$ be von Neumann algebras and $\phi_{i}$ faithful normal states on $M_{i}$. If $M_{1} \cap\left(M_{1}\right)_{\phi_{1}}^{\prime}=\mathbb{C}$, then $C_{\phi_{1} \otimes \phi_{2}}\left(M_{1} \bar{\otimes} M_{2}\right) \cap\left(M_{1}\right)_{\phi_{1}}^{\prime}=C_{\phi_{2}}\left(M_{2}\right)$. If moreover $M_{1}$ is a $\mathrm{III}_{1}$ factor, then $C_{\phi_{1} \otimes \phi_{2}}\left(M_{1} \bar{\otimes} M_{2}\right) \cap C_{\phi_{1}}\left(M_{1}\right)^{\prime}=\left(M_{2}\right)_{\phi_{2}}$.

Proof. Write $N:=C_{\phi_{1} \otimes \phi_{2}}\left(M_{1} \bar{\otimes} M_{2}\right)$. Since $N$ is contained in $M_{1} \bar{\otimes} M_{2} \bar{\otimes} \mathbb{B}\left(L^{2}(\mathbb{R})\right)$, $N \cap\left(M_{1}\right)_{\phi_{1}}^{\prime}$ is contained in $M_{1} \bar{\otimes} M_{2} \bar{\otimes} \mathbb{B}\left(L^{2}(\mathbb{R})\right) \cap\left(\left(M_{1}\right)_{\phi_{1}} \bar{\otimes} \mathbb{C} \bar{\otimes} \mathbb{C}\right)^{\prime}=\mathbb{C} \bar{\otimes} M_{2} \bar{\otimes} \mathbb{B}\left(L^{2}(\mathbb{R})\right)$. Hence it is contained in $\mathbb{C} \bar{\otimes} C_{\phi_{2}}\left(M_{2}\right)$ and the first statement holds. We then have

$$
N \cap C_{\phi_{1}}\left(M_{1}\right)^{\prime} \subset \mathbb{C} \bar{\otimes} C_{\phi_{2}}\left(M_{2}\right) \cap(\mathbb{C} \bar{\otimes} \mathbb{C} \bar{\otimes} L \mathbb{R})^{\prime}=\mathbb{C} \bar{\otimes}\left(M_{2}\right)_{\phi_{2}} \bar{\otimes} L \mathbb{R}
$$

(use [8, Proposition 2.4] if necessary). If $M_{1}$ is a $\mathrm{III}_{1}$ factor, exchanging the first and the second tensor components, we have

$$
\left(\left(M_{2}\right)_{\phi_{2}} \bar{\otimes} \mathbb{C} \bar{\otimes} L \mathbb{R}\right) \cap\left(\mathbb{C} \bar{\otimes} C_{\phi_{1}}\left(M_{1}\right)^{\prime}\right) \subset\left(M_{2}\right)_{\phi_{2}} \bar{\otimes}\left(C_{\phi_{1}}\left(M_{1}\right) \cap C_{\phi_{1}}\left(M_{1}\right)^{\prime}\right)=\left(M_{2}\right)_{\phi_{2}} \bar{\otimes} \mathbb{C} .
$$

By the first lemma, we identify $M$ as $N \bar{\otimes}\left(N^{\prime} \cap M\right)$ if $N \subset M$ satisfy the assumption of the lemma.

Let $\mathbb{G}_{i}$ and $N_{j}$ be as in the first statement in Theorem $\mathrm{A}$. For simplicity, we use the notation $M_{i}:=L^{\infty}\left(\mathbb{G}_{i}\right), M:=M_{1} \bar{\otimes} \cdots \bar{\otimes} M_{m}, N:=N_{1} \bar{\otimes} \cdots \bar{\otimes} N_{n}, M_{X}:=\bar{\otimes}_{i \in X} M_{i}\left(M_{\emptyset}:=\right.$ $\mathbb{C})$ and $N_{Y}:=\bar{\otimes}_{j \in Y} N_{j}\left(N_{\emptyset}:=\mathbb{C}\right)$ for any subsets $X \subset\{1, \ldots, m\}$ and $Y \subset\{1, \ldots, n\}$. Since the inclusion $N \subset M$ is with expectation, we have an inclusion $C(N) \subset C(M)$ for some continuous cores. Since each $N_{i}$ has an almost periodic state, there exist almost periodic weights $\phi_{i}$ such that $\left(N_{i}\right)_{\phi_{i}}$ is non-amenable and $\phi_{i}$ is semifinite on $\left(N_{i}\right)_{\phi_{i}}$. Write $\phi:=\phi_{1} \otimes \cdots \otimes \phi_{n}$. We fix an inclusion

$$
C_{\phi}(N) \simeq C(N) \subset C(M) \simeq C_{h}(M)
$$

which preserves canonical traces. We denote this trace by Tr. Since the inclusion does not preserve $L \mathbb{R}$, we write $L \mathbb{R}$ inside $C_{\phi}(N)$ and $C_{h}(M)$ as $L \mathbb{R}_{N}$ and $L \mathbb{R}_{M}$ respectively. Identifying $M_{X}=M_{X} \bar{\otimes} 1_{M_{X^{c}}}$ and $N_{Y}=N_{Y} \bar{\otimes} 1_{N_{Y^{c}}}$, we often write $C_{h}\left(M_{X}\right), C_{\phi}\left(N_{Y}\right)$, $\left(M_{X}\right)_{h}$, and $\left(N_{Y}\right)_{\phi}$ (instead of $C_{h_{X}}\left(M_{X}\right)$ for example, where $\left.h_{X}:=\otimes_{i \in X} h_{i}\right)$. 
Lemma 3.4.3. For any subset $Y \subset\{1, \ldots, n\}$ with $\left|Y^{c}\right| \leq m$ and any $\operatorname{Tr}$-finite projection $p \in L \mathbb{R}_{N}$, there are $X \subset\{1, \ldots, m\}$ and $\phi_{i}$-finite projections $p_{i} \in\left(N_{i}\right)_{\phi_{i}}$ such that $\left|X^{c}\right|=$ $\left|Y^{c}\right|$ and $q C_{\phi}\left(N_{Y}\right) q \preceq_{C(M)} C_{h}\left(M_{X}\right)$, where $q:=p_{1} \otimes \cdots \otimes p_{n} \otimes p$.

Proof. Let $z_{i} \in \mathcal{Z}\left(\left(N_{i}\right)_{\phi_{i}}\right)$ be projections such that $z_{i}\left(N_{i}\right)_{\phi_{i}}$ has no amenable summand. Since $\left(N_{i}\right)_{\phi_{i}}$ are semifinite, there are $\phi_{i}$-finite projections $p_{i} \in\left(N_{i}\right)_{\phi_{i}}$ such that $p_{i} z_{i} \neq 0$. Put $\tilde{p}_{i}:=p_{i} z_{i}, \tilde{p}:=\tilde{p}_{1} \otimes \cdots \otimes \tilde{p}_{n}, \tilde{N}_{i}:=\tilde{p}_{i} N_{i} \tilde{p}_{i}$, and $\tilde{N}:=\tilde{N}_{1} \bar{\otimes} \cdots \bar{\otimes} \tilde{N}_{n}$. For a Tr-finite projection $p \in L \mathbb{R}$, we have $p C_{\phi}(\tilde{N}) p=p \tilde{p} C_{\phi}(N) \tilde{p} p \subset C_{h}(M)$. We apply Proposition 3.2.1 to $p C_{\phi}\left(\tilde{N}_{2} \bar{\otimes} \cdots \bar{\otimes} \tilde{N}_{n}\right) p \subset C_{h}(M)$ and get $p C_{\phi}\left(\tilde{N}_{2} \bar{\otimes} \cdots \bar{\otimes} \tilde{N}_{n}\right) p \preceq C_{h}(M) C_{h}\left(M_{X_{1}}\right)$, where $X_{1}=\{1, \ldots, m\} \backslash i$ for some $i$, by non-amenability of $\left(\tilde{N}_{1}\right)_{\phi_{1}}$. For simplicity we assume $i=1$. Note that $\left(\tilde{N}_{n}\right)_{\phi}=\tilde{p}_{n}\left(N_{n}\right)_{\phi} \tilde{p}_{n}$ has no amenable summand and hence is of type II. By Corollary 2.3.7, there exist nonzero projections $e_{1} \in p\left(\tilde{N}_{n}\right)_{\phi} p, f_{1} \in C_{h}\left(M_{X_{1}}\right)$, and a partial isometry $V_{1} \in\left\langle C_{h}(M), C_{h}\left(M_{X_{1}}\right)\right\rangle$ such that $\operatorname{Tr}\left(f_{1}\right)<\infty, V_{1}^{*} V_{1} \in e_{1} C_{\phi}\left(\tilde{N}_{2} \bar{\otimes} \cdots \bar{\otimes} \tilde{N}_{n}\right)^{\prime}$, and $V_{1} V_{1}^{*}=f_{1} e_{X_{1}}$, where $e_{X_{1}}$ is the Jones projection for $C_{h}\left(M_{X_{1}}\right) \subset C_{h}(M)$. We have

$$
\begin{aligned}
V_{1} e_{1} C_{\phi}\left(\tilde{N}_{2} \bar{\otimes} \cdots \bar{\otimes} \tilde{N}_{n}\right) e_{1} V_{1}^{*} & \subset f_{1} e_{X_{1}}\left\langle C_{h}(M), C_{h}\left(M_{X_{1}}\right)\right\rangle f_{1} e_{X_{1}} \\
& =\mathbb{C} e_{M_{1}} \bar{\otimes} f_{1} C_{h}\left(M_{X_{1}}\right) f_{1},
\end{aligned}
$$

where $e_{M_{1}}$ is the projection from $L^{2}\left(M_{1}\right)$ onto $\mathbb{C} \hat{1}_{M_{1}}$. We again apply Proposition 3.2.1 to the inclusion and get $V_{1} e_{1} C_{\phi}\left(\tilde{N}_{3} \bar{\otimes} \cdots \bar{\otimes} \tilde{N}_{n}\right) e_{1} V_{1}^{*} \preceq \mathbb{C}_{e_{M_{1}} \bar{\otimes} C_{h}\left(M_{X_{1}}\right)} \mathbb{C} e_{M_{1}} \bar{\otimes} C_{h}\left(M_{X_{2}}\right)$ for some $X_{2}$ (since $\left(\tilde{N}_{2}\right)_{\phi}$ has no amenable summand). We assume $X_{2}=X_{1} \backslash\{2\}$. Then there exist nonzero projections $e_{2} \in e_{1}\left(\tilde{N}_{n}\right)_{\phi} e_{1}, f_{2} \in C_{h}\left(M_{X_{2}}\right)$, and a partial isometry $V_{2} \in \mathbb{C}_{M_{1}} \bar{\otimes}$ $\left\langle C_{h}\left(M_{X_{1}}\right), C_{h}\left(M_{X_{2}}\right)\right\rangle$ such that $\operatorname{Tr}\left(f_{2}\right)<\infty, V_{2}^{*} V_{2} \in V_{1} e_{2} V_{1}^{*}\left(V_{1} C_{\phi}\left(\tilde{N}_{3} \bar{\otimes} \cdots \bar{\otimes} \tilde{N}_{n}\right) V_{1}^{*}\right)^{\prime}$, $V_{2} V_{2}^{*}=\mathbb{C} e_{M_{1}} \bar{\otimes} f_{2} e_{X_{2}}^{X_{1}}$, where $e_{X_{2}}^{X_{1}}$ is the Jones projection for $C_{h}\left(M_{X_{2}}\right) \subset C_{h}\left(M_{X_{1}}\right)$. We get

$$
V_{2} V_{1} e_{2} C_{\phi}\left(\tilde{N}_{3} \bar{\otimes} \cdots \bar{\otimes} \tilde{N}_{n}\right) e_{2} V_{1}^{*} V_{2}^{*} \subset \mathbb{C} e_{M_{1}} \bar{\otimes} \mathbb{C} e_{M_{2}} \bar{\otimes} f_{2} C_{h}\left(M_{X_{2}}\right) f_{2},
$$

where $e_{M_{2}}$ is the projection from $L^{2}\left(M_{2}\right)$ onto $\mathbb{C} \hat{1}_{M_{2}}$. Since $V_{2} V_{1} \in\left\langle C_{h}(M), C_{h}\left(M_{X_{2}}\right)\right\rangle$, $\left(V_{2} V_{1}\right)^{*} V_{2} V_{1} \in e_{2} C_{\phi}\left(\tilde{N}_{3} \bar{\otimes} \cdots \bar{\otimes} \tilde{N}_{n}\right)^{\prime}$, and $V_{2} V_{1}\left(V_{2} V_{1}\right)^{*}=V_{2} V_{2}^{*}=e_{X_{2}} f_{2}$, where $e_{X_{2}}$ is the Jones projection for $C_{h}(M) \subset C_{h}\left(M_{X_{1}}\right)$, we have $p C_{\phi}\left(\tilde{N}_{3} \bar{\otimes} \cdots \bar{\otimes} \tilde{N}_{n}\right) p \preceq C_{h}(M) C_{h}\left(M_{X_{2}}\right)$. Hence $q C_{\phi}\left(N_{3} \bar{\otimes} \cdots \bar{\otimes} N_{n}\right) q \preceq C_{h}(M) C_{h}\left(M_{X_{2}}\right)$. Repeating this operation, we can prove the lemma.

Remark 3.4.4. In the proof of the lemma, we in fact proved the following statement: if $q C_{\phi}\left(N_{Y}\right) q \preceq_{C(M)} C_{h}\left(M_{X}\right)$ (resp. $q\left(N_{Y}\right)_{\phi} q \preceq_{C(M)} C_{h}\left(M_{X}\right)$ ) for some $X, Y$ and $q=$ $p_{1} \otimes \cdots \otimes p_{n} \otimes p$, where $p \in L \mathbb{R}_{N}$ is a Tr-finite projection and $p_{i} \in\left(N_{i}\right)_{\phi}$ are $\phi$-finite projections with $p_{i}\left(N_{i}\right)_{\phi} p_{i}$ non-amenable, then for any $i \in Y$ there is some $j \in X$ such that $q C_{\phi}\left(N_{Y \backslash\{i\}}\right) q \preceq_{C(M)} C_{h}\left(M_{X \backslash\{j\}}\right)$ (resp. $\left.q\left(N_{Y \backslash\{i\}}\right)_{\phi} q \preceq_{C(M)} C_{h}\left(M_{X \backslash\{j\}}\right)\right)$.

Corollary 3.4.5. If $q\left(N_{Y}\right)_{\phi} q \preceq_{C(M)} C_{h}\left(M_{X}\right)$ for some $X, Y$, where $q$ is as in the previous remark, then $|Y| \leq|X|$.

Proof. Suppose $|Y|>|X|$. By the remark above, we get $q\left(N_{\tilde{Y}}\right)_{\phi} q \preceq C_{h}(M) L \mathbb{R}$ for some $\tilde{Y} \neq \emptyset$. This is a contradiction since $q\left(N_{\tilde{Y}}\right)_{\phi} q$ has no amenable summand.

It is now easy to prove the first half of Theorem $\mathbb{\mathrm { A }}$, since $q C_{\phi}\left(N_{Y}\right) q \preceq_{C(M)} C_{h}\left(M_{X}\right)$ implies $q\left(N_{Y}\right)_{\phi} q \preceq_{C(M)} C_{h}\left(M_{X}\right)$.

We next assume $M_{i}=L^{\infty}\left(\mathbb{G}_{i}\right)$ and $N_{j}$ satisfy conditions in the second statement in Theorem A, In the case, $M$ is full from Lemmas 2.1.2 and 2.2.3, and hence each $N_{i}$ is also full. So $N_{i}=\left(N_{i}\right)_{\phi_{i}}$ is a non-amenable $\mathrm{II}_{1}$ factor if $N_{i}$ is a $\mathrm{II}_{1}$ factor. If $N_{i}$ is a $\mathrm{III}_{1}$ factor, as we mentioned in Subsection 2.1, $\phi_{i}$ is $\operatorname{Sd}\left(N_{i}\right)$-almost periodic and the discrete core $D_{\phi_{i}}\left(N_{i}\right)$ is a $\mathrm{II}_{\infty}$ factor and is isomorphic to $\left(N_{i}\right)_{\phi_{i}} \bar{\otimes} \mathbb{B}(H)$ for some $H$. Hence $\left(N_{i}\right)_{\phi_{i}}$ is 
a non-amenable $\mathrm{II}_{1}$ factor. Thus we can apply Lemma 3.4.3 and get that for any Tr-finite projection $p \in L \mathbb{R}_{N}$ and any $i$ there is some $j$ such that $p C_{\phi}\left(N_{i}\right) p \preceq_{C_{h}(M)} C_{h}\left(M_{j}\right)$. We first prove this correspondence is one to one. For simplicity, from now on we assume that there is at least one $\mathrm{III}_{1}$ factor among $M_{i}$.

Lemma 3.4.6. Let $X, Y$ be subsets in $\{1, \ldots, n\}$ and $p \in L \mathbb{R}_{N}$ a Tr-finite projection.

(i) If $N_{Y}$ is a $\mathrm{II}_{1}$ factor, then $\left(N_{Y}\right)_{\phi} p \preceq_{C_{h}(M)} C_{h}\left(M_{X}\right)$ implies $\left(N_{Y}\right)_{\phi} p \subset_{\text {approx }} C_{h}\left(M_{X}\right)$.

(ii) If $N_{Y}$ is a III $_{1}$ factor, then $p C_{\phi}\left(N_{Y}\right) p \preceq C_{h}(M) C_{h}\left(M_{X}\right)$ implies $p C_{\phi}\left(N_{Y}\right) p \subset_{\text {approx }}$ $C_{h}\left(M_{X}\right)$.

Proof. Use Lemmas 2.1.3, 2.4.10, and 3.4.2,

Lemma 3.4.7. Let $X_{1}, X_{2}$, and $Y$ be subsets in $\{1, \ldots, n\}$ with $\left|X_{1}\right|=\left|X_{2}\right|=|Y|$ and let $p_{1}, p_{2} \in L \mathbb{R}_{N}$ be Tr-finite projections. Assume that $p_{i}\left(N_{Y}\right)_{\phi} p_{i} \preceq_{C_{h}(M)} C_{h}\left(M_{X_{i}}\right)$ for $i=1,2$ when $N_{Y}$ is a $\mathrm{II}_{1}$ factor, or $p_{i} C_{\phi}\left(N_{Y}\right) p_{i} \preceq_{C_{h}(M)} C_{h}\left(M_{X_{i}}\right)$ for $i=1,2$ when $N_{Y}$ is $a \mathrm{III}_{1}$ factor. Then $X_{1}=X_{2}$.

Proof. Replacing $p_{1}$ and $p_{2}$ with $p_{1} \vee p_{2}$, we may assume $p_{1}=p_{2}(=: p)$. By the previous lemma, we have $\left(N_{Y}\right)_{\phi} p \subset_{\text {approx }} C_{h}\left(M_{X_{a}}\right)$ for $a=1,2$. Lemma 2.4.8 (and Remark 2.4.9) then implies $\left(N_{Y}\right)_{\phi} p \subset_{\text {approx }} C_{h}\left(M_{X_{1} \cap X_{2}}\right)$. Thus we get $\left(N_{Y}\right)_{\phi} p \preceq_{C_{h}(M)} C_{h}\left(M_{X_{1} \cap X_{2}}\right)$. This contradicts to Corollary 3.4 .5 when $X_{1} \neq X_{2}$, since $\left|X_{1} \cap X_{2}\right|<|Y|$.

Lemma 3.4.8. Let $X, Y_{1}$, and $Y_{2}$ be subsets in $\{1, \ldots, n\}$ with $|X|=\left|Y_{1}\right|=\left|Y_{2}\right|$ and let $p_{1}, p_{2} \in L \mathbb{R}_{N}$ be Tr-finite projections. If $p_{1} C_{\phi}\left(N_{Y_{1}}\right) p_{1} \preceq_{C_{h}(M)} C_{h}\left(M_{X}\right)$ and $p_{2} C_{\phi}\left(N_{Y_{2}}\right) p_{2} \preceq_{C_{h}(M)}$ $C_{h}\left(M_{X}\right)$, then $Y_{1}=Y_{2}$.

Proof. Replacing $p_{1}$ and $p_{2}$ with $p_{1} \vee p_{2}$, we may assume $p_{1}=p_{2}(=: p)$. By Lemma 3.4.3. there is some $X_{2}$ with $\left|X_{2}\right|=\left|Y_{1}^{c}\right|$ such that $p C_{\phi}\left(N_{Y_{1}^{c}}\right) p \preceq_{C_{h}(M)} C_{h}\left(M_{X_{2}}\right)$. We claim that $X \cap X_{2} \neq \emptyset$ when $Y_{1} \neq Y_{2}$. Take $i \in Y_{1}^{c} \cap Y_{2}$. Since $i \in Y_{1}^{c}$, by Remark 3.4.2, we have $p C_{\phi}\left(N_{i}\right) p \preceq_{C_{h}(M)} C_{h}\left(M_{j}\right)$ for some $j \in X_{2}$. Since $i \in Y_{2}$, we also have $p C_{\phi}\left(N_{i}\right) p \preceq_{C_{h}(M)} C_{h}\left(M_{l}\right)$ for some $l \in X$ by Remark 3.4 .2 and assumption. By the previous lemma, $j=l \in X \cap X_{2}$.

By assumption, there is $q \in L \mathbb{R}_{M}$ such that $p C_{\phi}\left(N_{Y_{1}}\right) p \preceq_{r C_{h}(M) r} q C_{h}\left(M_{X}\right) q$ for $r:=$ $p \vee q$. By [26, Lemma 3.5] and Lemma 3.4.2, we have

$$
q C_{h}\left(M_{X}\right)^{\prime} q \cap q C_{h}(M) q \preceq_{r} C_{h}(M) r p C_{\phi}\left(N_{Y_{1}}\right)^{\prime} p \cap p C_{h}(M) p
$$

and

$$
\left(M_{X^{c}}\right)_{h} q \subset q C_{h}\left(M_{X}\right)^{\prime} q \preceq p C_{\phi}\left(N_{Y_{1}}\right)^{\prime} p \subset p C_{\phi}\left(N_{Y_{1}^{c}}\right) p \preceq q C_{h}\left(M_{X_{2}}\right) q .
$$

When $N_{Y_{1}^{c}}$ is a $\mathrm{III}_{1}$ factor, the final embedding becomes $C_{\text {approx }}$. When $N_{Y_{1}^{c}}$ is a $\mathrm{II}_{1}$ factor, since $N_{Y_{1}}$ is a $\mathrm{III}_{1}$ factor, we have $C_{\phi}\left(N_{Y_{1}}\right)^{\prime}=\left(N_{Y_{1}^{c}}\right)_{\phi}$ and hence replacing the final embedding with $\left(N_{Y_{1}^{c}}\right)_{\phi} p \preceq q C_{h}\left(M_{X_{2}}\right) q$, we again get $\subset_{\text {approx }}$. In any case, by [26. Lemma 3.8], we get $\left(M_{X^{c}}\right)_{h} q \preceq_{C_{h}(M)} C_{h}\left(M_{X_{2}}\right)$. By Lemma 2.4.6, there is some $s \in\left(M_{X^{c}}\right)_{h}^{\prime} \cap q C_{h}(M) q$ such that $\left(M_{X^{c}}\right)_{h} q s C_{\text {approx }} C_{h}\left(M_{X_{2}}\right)$. Since we trivially have $\left(M_{X^{c}}\right)_{h} q \subset$ approx $C_{h}\left(M_{X^{c}}\right)$, it also holds that $\left(M_{X^{c}}\right)_{h} q s \subset_{\text {approx }} C_{h}\left(M_{X^{c}}\right)$ by Lemma 2.4.3. Finally from Remark 2.4.10, we get $\left(M_{X^{c}}\right)_{h} q s C_{\text {approx }} C_{h}\left(M_{X^{c} \cap X_{2}}\right)$. Since $\left|X^{c} \cap X_{2}\right|<\left|X^{c}\right|$ by the claim above, we get a contradiction from Corollary 3.4.5.

Thanks for previous two lemmas, there is a unique $\sigma \in \mathfrak{S}_{n}$ such that

$$
p C_{\phi}\left(N_{i}\right) p \preceq_{C_{h}(M)} C_{h}\left(M_{\sigma(i)}\right) \quad(i=1, \ldots, n)
$$


for any $\operatorname{Tr}$-finite projection $p \in L \mathbb{R}_{N}$. In this case, we in fact have

$$
p C_{\phi}\left(N_{Y}\right) p \preceq_{C_{h}(M)} C_{h}\left(M_{\sigma(Y)}\right) \quad(Y \subset\{1, \ldots, n\}) .
$$

To see this, fix any $Y \subset\{1, \ldots, n\}$. We use Lemma 3.4 .3 and find some $X \subset\{1, \ldots, n\}$, which is unique by Lemma 3.4.7, such that $p C_{\phi}\left(N_{Y}\right) p \preceq_{C_{h}(M)} C_{h}\left(M_{X}\right)$ and $|X|=|Y|$. By Remark 3.4.4, for any $i \in Y$ there is $j \in X$ such that $p C_{\phi}\left(N_{i}\right) p \preceq_{C_{h}(M)} C_{h}\left(M_{j}\right)$. This implies $\sigma(i)=j$ and, since $\sigma$ is bijective and $|X|=|Y|$, we get $\sigma(Y)=X$.

We next take the relative commutants of the embedding (with respect to $Y^{c}$ ) and get

$$
\left(M_{\sigma(Y)}\right)_{h} q \subset q C_{h}\left(M_{\sigma\left(Y^{c}\right)}\right)^{\prime} q \preceq C_{h}(M) C_{\phi}\left(N_{Y^{c}}\right)^{\prime} \subset\left(N_{Y}\right)_{\phi} \bar{\otimes} L \mathbb{R}
$$

for some Tr-finite projection $q \in L \mathbb{R}_{M}$. Then by (the proof of) [1, Proposition 2.10], we finally get

$$
\left(M_{\sigma(Y)}\right)_{h} \preceq_{M}\left(N_{Y}\right)_{\phi} \quad(Y \subset\{1, \ldots, n\}) .
$$

Lemma 3.4.9. In the setting, $M_{\sigma(i)}$ is a $\mathrm{II}_{1}$ factor if and only if so is $N_{i}$.

Proof. Suppose that $M_{\sigma(i)}$ is a $\mathrm{II}_{1}$ factor. Then since

$$
\left(N_{i}\right)_{\phi} \bar{\otimes} L \mathbb{R} p \subset p C_{\phi}\left(N_{i}\right) p \preceq_{C_{h}(M)} C_{h}\left(M_{\sigma(i)}\right)=\left(M_{\sigma(i)}\right)_{h} \bar{\otimes} L \mathbb{R}
$$

for any Tr-finite projection $p \in L \mathbb{R}_{N}$, we have $\left(N_{i}\right)_{\phi} \preceq_{M}\left(M_{\sigma(i)}\right)_{h}$ by [1, Proposition 2.10]. If $N_{i}$ is of type III, we can apply Lemma 2.3.10 two times and get

$$
N_{i}=\left(\left(N_{i}\right)_{\phi}^{\prime} \cap M\right)^{\prime} \cap M \preceq_{M}\left(\left(M_{\sigma(i)}\right)_{h}^{\prime} \cap M\right)^{\prime} \cap M=M_{\sigma(i)} .
$$

Hence $N_{i}$ must contains a finite direct summand and hence a contradiction. Since $\left(M_{\sigma(i)}\right)_{h} \preceq_{M}$ $\left(N_{i}\right)_{\phi}$, the converse holds by the same argument.

Proof of Theorem A. The first half statement was already proved by Corollary 3.4.5. So we see the second half.

\section{- Case 1: all $\mathrm{M}_{\mathbf{i}}$ are $\mathrm{II}_{1}$ factors.}

In the case, we can apply the prime factorization result of Ozawa and Popa. So there are $u \in \mathcal{U}(M), \sigma \in \mathfrak{S}_{n}$, and $t_{i}>0$ such that $u N_{i} u^{*}=M_{\sigma(i)}^{t_{i}}$ (which implies $\left.N_{i} \preceq_{M} M_{\sigma(i)}\right)$. By the same method as in Lemmas 3.4.7 and 3.4.8, we can prove that $\sigma \in \mathfrak{S}_{n}$ is unique with the condition $N_{i} \preceq_{M} M_{\sigma(i)}$ for all $i$. On the other hand, by Lemma 3.4.3, for any $i$ there is some $j$ such that $N_{i} \bar{\otimes} L \mathbb{R} p=p C_{\phi}\left(N_{i}\right) p \preceq C_{h}(M) C_{h}\left(M_{j}\right)=M_{j} \bar{\otimes} L \mathbb{R}$ for any Tr-finite projection $p \in L \mathbb{R}_{N}$. By [1, Proposition 2.10], this implies $N_{i} \preceq_{M} M_{j}$. So by the uniqueness, we get $\sigma(i)=j$ and $\sigma$ is determined from $p C_{\phi}\left(N_{i}\right) p \preceq_{C_{h}(M)} C_{h}\left(M_{\sigma(i)}\right)$.

We next consider the case that there is at least one $\mathrm{III}_{1}$ factor among $M_{i}$. As we mentioned before Lemma 3.4.9, there is a unique $\sigma \in \mathfrak{S}_{n}$ with $p C_{\phi}\left(N_{i}\right) p \preceq_{C_{h}(M)} C_{h}\left(M_{\sigma(i)}\right)$, and it then holds that $\left(M_{\sigma(i)}\right)_{h} \preceq_{M}\left(N_{i}\right)_{\phi}$.

- Case 2: there are more than two $\mathrm{III}_{1}$ factors among $\mathbf{M}_{\mathbf{i}}$.

By Lemma 3.4.9, the same is true for $N_{i}$. For simplicity we assume $\sigma=$ id. When $M_{i}$ and $N_{i}$ are III $_{1}$ factors, since so are $M_{\{i\}^{c}}$ and $N_{\{i\}^{c}}$, we can apply Lemma 2.3.10 to $\left(M_{i}\right)_{h} \preceq_{M}\left(N_{i}\right)_{\phi}$ two times and get $M_{i} \preceq_{M} N_{i}$. Also $M_{i} \preceq_{M} N_{i}$ holds for $\mathrm{II}_{1}$ factors $M_{i}$ and $N_{i}$. We fix $i$ and apply Lemma 3.3 .2 and get a partial isometry or a unitary $u \in M$ such that $u M_{i} u^{*} \subset u u^{*} N_{i} u u^{*}$. Write $p=u u^{*} \in N_{i}$ and $q=u^{*} u \in M_{i}$. We have

$$
u M_{i} u^{*} \bar{\otimes} u M_{\{i\} c} u^{*}=u M u^{*}=p M p=p N_{i} p \bar{\otimes} N_{\{i\}^{c}},
$$


and hence

$$
u M_{\{i\}^{c}} u^{*}=\left(u M_{i} u^{*}\right)^{\prime} \cap p M p=\left(\left(u M_{i} u^{*}\right)^{\prime} \cap p N_{i} p\right) \bar{\otimes} N_{\{i\}^{c}} .
$$

Since $M_{i} \simeq u M_{i} u^{*}$, we can apply the first half of Theorem $\mathrm{A}$, and then get amenability of $\left(\left(u M_{i} u^{*}\right)^{\prime} \cap p N_{i} p\right)$. Since $M_{\{i\}^{c}}$ is full from Lemmas 2.1.2 and 2.2.3, $\left(\left(u M_{i} u^{*}\right)^{\prime} \cap p N_{i} p\right)$ must be a type I factor, say $\mathbb{B}(K)$. Since $u M u^{*}=u M_{i} u^{*} \bar{\otimes} u M_{\{i\}} u^{*}=u M_{i} u^{*} \bar{\otimes} \mathbb{B}(K) \bar{\otimes} N_{\{i\}^{c}}$, we get $p N_{i} p=\left(N_{\{i\}^{c}}\right)^{\prime} \cap p M p=u M_{i} u^{*} \bar{\otimes} \mathbb{B}(K)$. This means $N_{i}$ and $M_{i}$ are isomorphic when they are $\mathrm{III}_{1}$ factors, and are stably isomorphic when they are $\mathrm{II}_{1}$ factors.

\section{- Case 3: there is only one $\mathrm{III}_{1}$ factor among $\mathrm{M}_{\mathbf{i}}$.}

The same is true for $N_{i}$. Assume for simplicity that $\sigma=\mathrm{id}$ and $M_{1}$ and $N_{1}$ are $\mathrm{III}_{1}$ factors. For $i \neq 1$, since $M_{i} \preceq_{M} N_{i}, M_{i}$ and $N_{i}$ are stably isomorphic by the same manner as above. So we see the case $i=1$. Put $X:=\{1\}^{c}$. Since $N_{X}$ and $M_{X}$ are also $\mathrm{II}_{1}$ factors, we have $M_{X}=\left(M_{X}\right)_{h} \preceq_{M}\left(N_{X}\right)_{\phi}=N_{X}$. So there is a partial isometry $u \in M$ such that $u M_{X} u^{*} \subset p N_{X} p$, where $p:=u u^{*}$. We have $u M_{1} u^{*}=\left(\left(u M_{X} u^{*}\right)^{\prime} \cap p N_{X} p\right) \bar{\otimes} N_{1}$. The algebra $\left(u M_{X} u^{*}\right)^{\prime} \cap p N_{X} p$ must be a type I factor and hence $M_{1}$ and $N_{1}$ are isomorphic.

\section{Prime factorization results for crossed product algebras}

In the proof of Theorem $\mathrm{B}$, we go along a similar line to the proof of Theorem $\mathrm{A}$. We first prove a key proposition with condition $(\mathrm{AO})$ and then study intertwiners on crossed products. We finally prove some one-to-one correspondence between each component of direct product groups.

\subsection{Location of subalgebras}

Let $\Gamma$ and $\Lambda$ be discrete groups acting on semifinite tracial von Neumann algebras $\left(A, \operatorname{Tr}_{A}\right)$ and $\left(B, \tau_{B}\right)$ with $\tau_{B}(1)=1$ as trace preserving actions. Assume that we have an inclusion $B \rtimes \Lambda \subset p(A \rtimes \Gamma) p$ with $B \subset p A p$ (not necessarily $B=p A p$ ) for some $\operatorname{Tr}_{A}$-finite projection $p \in \mathcal{Z}(A)$. Write $M:=A \rtimes \Gamma$.

Lemma 4.1.1. Let $\Lambda_{0} \subset \Lambda$ and $\Gamma_{0} \subset \Gamma$ be subgroups and $q \in \mathcal{Z}(A)$ a $\operatorname{Tr}_{A}$-finite projection with $p \leq q$. If $L \Lambda_{0} \preceq_{q M q} q\left(A \rtimes \Gamma_{0}\right) q$, then we have $B \rtimes \Lambda_{0} \preceq_{q M q} q\left(A \rtimes \Gamma_{0}\right) q$.

Proof. By assumption, there exist $\delta>0$ and a finite subset $\mathcal{F} \subset p M q$ such that $\sum_{x, y \in \mathcal{F}}\left\|E_{A \rtimes \Gamma_{0}}\left(y^{*} w x\right)\right\|_{2}^{2}>\delta$ for all $w \in \mathcal{U}\left(L \Lambda_{0}\right)$. We may assume that $\mathcal{F}$ consists of elements of the form $p \lambda_{s} q$ for some $s \in \Gamma$. Put $d_{0}:=\sum_{x \in \mathcal{F}} x e_{A \rtimes \Gamma_{0}} x^{*}$. By the proof of (i) $\Rightarrow$ (ii) in Proposition 2.3.5, $d_{0}$ satisfies condition (ii) in the proposition. Since $d_{0}$ commutes with $B \subset p A p, d_{0}$ actually satisfies that the $\sigma$-weak closure of

$$
\operatorname{co}\left\{w d_{0} w^{*} \mid w=\lambda_{s} b \text { for some } s \in \Lambda_{0}, b \in \mathcal{U}(B)\right\}
$$

does not contain zero. Then by the same manner as in the proof of (ii) $\Rightarrow$ (iii) in Proposition 2.3.5, we get $d \in\left\langle M, A \rtimes \Gamma_{0}\right\rangle \cap\left(L \Lambda_{0} \cup B\right)^{\prime}$ satisfying condition (iii). Since $\left(L \Lambda_{0} \cup B\right)^{\prime}=\left(B \rtimes \Lambda_{0}\right)^{\prime}$, we get $B \rtimes \Lambda_{0} \preceq_{q M q} q\left(A \rtimes \Gamma_{0}\right) q$.

Proposition 4.1.2. Assume that $\operatorname{Tr}_{A} \mid \mathcal{Z}(A)$ is semifinite and $\Gamma$ is bi-exact relative to $\mathcal{G}$, where $\mathcal{G}$ is a countable family of subgroups of $\Gamma$. Assume either $A$ is amenable or $\Gamma$ is weakly amenable. Then for any subgroup $\Lambda_{0} \subset \Lambda$, we have either one of the following conditions:

(i) There is a conditional expectation from $p\langle M, A\rangle p$ onto $L \Lambda_{0}^{\prime} \cap p M p$, which restricts to the trace preserving expectation on $p M p$. 
(ii) There exists $\Gamma_{0} \in \mathcal{G}$ and $a \operatorname{Tr}_{A}$-finite projection $q \in \mathcal{Z}(A)$ with $p \leq q$ such that $B \rtimes \Lambda_{0} \preceq_{q M q} q\left(A \rtimes \Gamma_{0}\right) q$.

Proof. Since $\left.\operatorname{Tr}_{A}\right|_{\mathcal{Z}(A)}$ is semifinite, condition (ii) is equivalent to that there exists $\Gamma_{0} \in \mathcal{G}$ such that $B \rtimes \Lambda_{0} \preceq_{M} A \rtimes \Gamma_{0}$.

Suppose that $A$ is amenable. Then since $p\langle M, A\rangle p$ is also amenable, condition (i) means amenability of $L \Lambda_{0}^{\prime} \cap p M p$. Assume that $L \Lambda_{0}^{\prime} \cap p M p$ is non-amenable. Then by [11, Theorem 5.3.3 and Remark 5.3.4], there exists $\Gamma_{0} \in \mathcal{G}$ and a projection $q \in \mathcal{Z}(A)$ with $\operatorname{Tr}_{A}(q)<\infty$ such that $L \Lambda_{0} \preceq_{r M r} q\left(A \rtimes \Gamma_{0}\right) q$, where $r:=p \vee q$. Exchanging $r$ with $q$, we may assume $p \leq q$ and $r=q$. By the last lemma, we get $B \rtimes \Lambda_{0} \preceq_{q M q} q\left(A \rtimes \Gamma_{0}\right) q$.

Suppose next $\Gamma$ is weakly amenable and $B \rtimes \Lambda_{0} \npreceq_{M} A \rtimes \Gamma_{0}$ for any $\Gamma_{0} \in \mathcal{G}$. This means $L \Lambda_{0} \npreceq_{M} A \rtimes \Gamma_{0}$ for any $\Gamma_{0} \in \mathcal{G}$ by the previous lemma. Then by [11, Proposition 5.2.4], there is a unital diffuse abelian subalgebra $A_{0} \subset L \Lambda_{0}$ such that $A_{0} \npreceq_{M} A \rtimes \Gamma_{0}$ for any $\Gamma_{0} \in \mathcal{G}$. By [20, Theorem 3.1], there is a conditional expectation from $p\langle M, A\rangle p$ onto $\mathcal{N}_{p M p}\left(A_{0}\right)^{\prime \prime}$, which restricts to the trace preserving expectation on $p M p$. Since $\mathcal{N}_{p M p}\left(A_{0}\right)^{\prime \prime}$ contains $L \Lambda_{0}^{\prime} \cap p M p$, we are done.

\subsection{Intertwiners inside crossed product von Neumann algebras}

In the subsection, we study intertwiners in crossed product von Neumann algebras. Under the assumption of freeness of actions, we can construct a special form of an embedding and an intertwiner.

Lemma 4.2.1. Let $A$ be a commutative von Neumann algebra and $p_{i} \in A(i \in \mathbb{N})$ be projections. If $\sum_{i} p_{i} \leq n$ for some $n \in \mathbb{N}$, then there exists a projection $z \in A$ such that $\sum_{i} z p_{i} \neq 0$ and $z p_{i}=0$ except for finitely many $i$.

Proof. If $p_{1}$ is orthogonal to any other $p_{i}$, then $p_{1}$ does the work. If not, then we find the minimum number $i_{2}$ in the set $\left\{j \mid p_{1} p_{j} \neq 0, j \neq 1\right\}$. If $p_{1} p_{i_{2}}$ is orthogonal to any other $p_{i}$, then $p_{1} p_{i_{2}}$ does the work. Repeating this argument, we get $p_{1} p_{i_{2}} \cdots p_{i_{m}}(m \leq n)$ which is orthogonal to any other $p_{i}$. In fact, if we have a non-zero projection $p_{1} p_{i_{2}} \cdots p_{i_{m}}$ for $n+1 \leq m$, then we have $m \cdot p_{1} p_{i_{2}} \cdots p_{i_{m}} \leq p_{1}+p_{i_{2}}+\cdots+p_{i_{m}} \leq n$. Hence a contradiction.

Lemma 4.2.2. Let $\Gamma$ be a discrete group and $\alpha$ a free action on a commutative von Neumann algebra $A$. For any non-zero projection $p \in A$ and any distinct elements $s_{1}, \ldots, s_{n} \in \Gamma$, there is a non-zero projection $q \in A$ such that $q \leq p$ and $\alpha_{s_{i}}(q) \alpha_{s_{j}}(q)=0$ for any $i, j$ with $i \neq j$.

Proof. Write $A=L^{\infty}(X, \mu)$ for some standard probability space $(X, \mu)$. We may assume that $\Gamma$ acts on $(X, \mu)$ as a free action. Put $X_{g}:=\{x \in X \mid g \cdot x=x\}$ for any $g \in \Gamma$. Then by freeness, we have $\mu\left(X_{s_{i}^{-1} s_{j}}\right)=0$ for $i, j$ with $i \neq j$. Hence we have $\mu\left(\cup_{i \neq j} X_{s_{i}^{-1} s_{j}}\right)=0$. This implies $Y \cap\left(\cup_{i \neq j} X_{s_{i}^{-1} s_{j}}\right)^{c}$ is non-null for any non-null set $Y \subset X$.

Since $X$ is a standard Borel space, it is isomorphic to $\mathbb{R}$ (or a countable set). Let $E_{k}$ $\left(k \in \mathbb{N}\right.$ ) be a countable basis of open subsets of $\mathbb{R}$ (or the countable set). Let $E_{k} \subset X$ $(k \in \mathbb{N})$ be Borel subsets satisfying for any $x_{1}, \ldots, x_{n^{2}} \in X$ with $x_{1} \neq x_{i}$ for any $i \neq 1$, there exists $E_{k}$ such that $x_{1} \in E_{k}$ and $x_{i} \notin E_{k}$ for any $i \neq 1$ (e.g. take a basis of open subsets of $\mathbb{R}$ ). Then we have

$$
\begin{aligned}
\left(\cup_{i \neq j} X_{s_{i}^{-1} s_{j}}\right)^{c} & =\cap_{i \neq j} X_{s_{i}^{-1} s_{j}}^{c} \\
& =\left\{x \in X \mid s_{i}^{-1} s_{j} \cdot x \neq x \text { for any } i \neq j\right\} \\
& =\cup_{k}\left(E_{k} \backslash\left(\cup_{i \neq j} s_{j}^{-1} s_{i} E_{k}\right)\right) .
\end{aligned}
$$


Let $Y \subset X$ be a non-null set whose characteristic function is $p$. Then we can find some $k$ such that $Y \cap\left(E_{k} \backslash\left(\cup_{i \neq j} s_{j}^{-1} s_{i} E_{k}\right)\right)$ is non-null. Let $q$ be the projection corresponding to this set. Then it satisfies $q \leq p$ and $\alpha_{s_{j}^{-1} s_{i}}(q) q=0$ for $i \neq j$.

Lemma 4.2.3. Let $M$ be a $\mathrm{II}_{1}$ factor and $A \subset M$ be a regular von Neumann subalgebra (namely, $\mathcal{N}_{M}(A)^{\prime \prime}=M$ ). If non-zero projections e, $f \in \mathcal{Z}(A)$ have the same trace in $M$, then there exists $u \in \mathcal{N}_{M}(A)$ such that ueu* $=f$.

Proof. See the proof of [2, Lemma F.16].

Lemma 4.2.4. Let $\Gamma$ be a discrete group and $\alpha$ an action on a von Neumann algebra $A$. Let $\Gamma_{0}$ be a (possibly trivial) subgroup of $\Gamma$ and $\sigma: \Gamma / \Gamma_{0} \rightarrow \Gamma$ a section with $\sigma\left(\Gamma_{0}\right)=e$, where $e$ is the unit of $\Gamma$. Consider the unitary element

$$
U_{\sigma}: L^{2}(A) \otimes \ell^{2}(\Gamma) \rightarrow L^{2}(A) \otimes \ell^{2}\left(\Gamma_{0}\right) \otimes \ell^{2}\left(\Gamma / \Gamma_{0}\right) ; \hat{a} \otimes \delta_{\sigma\left(s \Gamma_{0}\right) g} \mapsto \hat{a} \otimes \delta_{g} \otimes \delta_{s \Gamma_{0}} .
$$

Then $U_{\sigma}$ gives an identification

$$
\operatorname{Ad} U_{\sigma}:\left\langle A \rtimes \Gamma, A \rtimes \Gamma_{0}\right\rangle \stackrel{\sim}{\rightarrow}\left(A \rtimes \Gamma_{0}\right) \bar{\otimes} \mathbb{B}\left(\ell^{2}\left(\Gamma / \Gamma_{0}\right)\right) .
$$

We have $\operatorname{Ad} U_{\sigma}(a)=\sum_{s \Gamma_{0} \in \Gamma / \Gamma_{0}} \alpha_{\sigma\left(s \Gamma_{0}\right)}^{-1}(a) \otimes e_{s \Gamma_{0}}$ for $a \in A$, where $e_{s \Gamma_{0}}$ is the orthogonal projection onto $\mathbb{C} \delta_{s \Gamma_{0}}$. If the action $\alpha$ is free on $A$, we have

$$
\operatorname{Ad} U_{\sigma}: A^{\prime} \cap\left\langle A \rtimes \Gamma, A \rtimes \Gamma_{0}\right\rangle \stackrel{\sim}{\rightarrow} \mathcal{Z}(A) \bar{\otimes} \ell^{\infty}\left(\Gamma / \Gamma_{0}\right) .
$$

Proof. Since the right action of $A \rtimes \Gamma_{0}$ satisfies $U_{\sigma} x^{\mathrm{op}} U_{\sigma}^{*}=x^{\mathrm{op}} \bar{\otimes} 1_{\mathbb{B}\left(\ell^{2}\left(\Gamma / \Gamma_{0}\right)\right)}$ for $x \in A \rtimes \Gamma_{0}$, we have

$$
\begin{aligned}
U_{\sigma}\left\langle A \rtimes \Gamma, A \rtimes \Gamma_{0}\right\rangle U_{\sigma}^{*}=U_{\sigma}\left(\left(A \rtimes \Gamma_{0}\right)^{\mathrm{op}}\right)^{\prime} U_{\sigma}^{*} & =\left(\left(A \rtimes \Gamma_{0}\right)^{\mathrm{op}}\right)^{\prime} \bar{\otimes} \mathbb{B}\left(\ell^{2}\left(\Gamma / \Gamma_{0}\right)\right) \\
& =\left(A \rtimes \Gamma_{0}\right) \bar{\otimes} \mathbb{B}\left(\ell^{2}\left(\Gamma / \Gamma_{0}\right)\right) .
\end{aligned}
$$

The formula of $\operatorname{Ad} U_{\sigma}(a)$ for $a \in A$ follows from a direct calculation.

Next assume that the action is free. Let $x$ be in $\left(A \rtimes \Gamma_{0}\right) \bar{\otimes} \mathbb{B}\left(\ell^{2}\left(\Gamma / \Gamma_{0}\right)\right)$. Write it as $x=\sum_{s, t \in \Gamma / \Gamma_{0}} x_{s, t} \otimes e_{s, t}$, where $x_{s, t} \in A \rtimes \Gamma_{0}$ and $e_{s, t}$ is the matrix unit along $s, t \in \Gamma / \Gamma_{0}$. By the first part of the statement, elements of $A$ in $\left(A \rtimes \Gamma_{0}\right) \bar{\otimes} \mathbb{B}\left(\ell^{2}\left(\Gamma / \Gamma_{0}\right)\right)$ is of the form $a=\sum_{s \in \Gamma / \Gamma_{0}} a_{s, s} \otimes e_{s, s}$, where $a_{s, s}=\alpha_{\sigma(s)}^{-1}(a)$. If $x$ commutes $a \in A$, a simple calculation shows that $x_{s, t} a_{t, t}=a_{s, s} x_{s, t}$ for any $s, t \in \Gamma / \Gamma_{0}$.

When $s=t$, then we get $x_{s, s} \alpha_{\sigma(s)}^{-1}(a)=\alpha_{\sigma(s)}^{-1}(a) x_{s, s}$ for any $a \in A$. Hence by the freeness, we get $x_{s, s} \in A^{\prime} \cap\left(A \rtimes \Gamma_{0}\right)=\mathcal{Z}(A)$. When $s \neq t$, then we have $x_{s, t} \alpha_{\sigma(t)}^{-1}(a)=$ $\alpha_{\sigma(s)}^{-1}(a) x_{s, t}$ for any $a \in A$. This means $x_{s, t} a=\alpha_{k}^{-1}(a) x_{s, t}$ for all $a \in A$, where $k=$ $\sigma(t)^{-1} \sigma(s) \neq e$. Let $x_{s, t}=\sum_{b \in \Gamma_{0}} \lambda_{b}\left(x_{s, t}\right)_{b}$ be the Fourier expansion of $x_{s, t}$ in $A \rtimes \Gamma_{0}$. Then the equality means $\left(x_{s, t}\right)_{b} a=\alpha_{k b}^{-1}(a)\left(x_{s, t}\right)_{b}$ for any $b \in \Gamma_{0}$ and $a \in A$. Since $k b$ is not the unit of $\Gamma$, we get $\left(x_{s, t}\right)_{b}=0$ for $b \in \Gamma_{0}$ and hence $x_{s, t}=0$. Thus $x$ is contained in $\mathcal{Z}(A) \bar{\otimes} \ell^{\infty}\left(\Gamma / \Gamma_{0}\right)$.

From now on, we keep the following setting. Let $\Gamma$ be a discrete group and $\alpha$ a trace preserving free action of $\Gamma$ on a semifinite tracial von Neumann algebra $\left(A, \operatorname{Tr}_{A}\right)$ with $\left.\operatorname{Tr}_{A}\right|_{\mathcal{Z}(A)}$ semifinite. Write $M:=A \rtimes \Gamma$. Let $\Gamma_{0} \subset \Gamma$ be a (possibly trivial) subgroup. Let $p \in \mathcal{Z}(A)$ be a projection with $\operatorname{Tr}_{A}(p)=1$ and $N \subset p(A \rtimes \Gamma) p$ a von Neumann subalgebra containing $p A p$. Assume either that $\mathcal{Z}(A)$ is diffuse, or $A$ is a $\mathrm{II}_{1}$ factor (so that $\operatorname{Tr}_{A}$ is finite and $\left.p=1_{A}\right)$. We fix $U_{\sigma}$ in the previous lemma for a section $\sigma$ and write $\tilde{s}:=\sigma(s)$ for $s \in \Gamma / \Gamma_{0}$. 
Proposition 4.2.5. If $N \preceq_{M} A \rtimes \Gamma_{0}$, then there exist non-zero projections $e \in \mathcal{Z}(A) p$ and $d \in(N)^{\prime} e \cap\left\langle M, A \rtimes \Gamma_{0}\right\rangle$ such that $U_{\sigma} d U_{\sigma}^{*}$ is contained in $\left(\mathbb{C} \bar{\otimes} \ell^{\infty}(\mathcal{S})\right) U_{\sigma} e U_{\sigma}^{*}$ for a finite subset $\mathcal{S} \subset \Gamma / \Gamma_{0}$. In the case, choosing an appropriate $\mathcal{S}$, a*-homomorphism $\pi: e N e \ni x \mapsto U_{\sigma} d x U_{\sigma}^{*} \in\left(A \rtimes \Gamma_{0}\right) \bar{\otimes} \mathbb{B}\left(\ell^{2}(\mathcal{S})\right)$ satisfies that $\pi(e A e) \subset A \bar{\otimes} \ell^{\infty}(\mathcal{S})$ and $\pi(a)=\sum_{s \in \mathcal{S}} \alpha_{\tilde{s}}^{-1}(a) \otimes e_{s, s}$ for $a \in e A e$.

Proof. By assumption there is an element $d \in N^{\prime} \cap\left\langle M, A \rtimes \Gamma_{0}\right\rangle$ satisfying $d=d p$ and $\operatorname{Tr}_{\left\langle M, A \rtimes \Gamma_{0}\right\rangle}(d)<\infty$. Taking a spectral projection of $d$, we may assume $d$ is a projection. By the last lemma, $d$ is contained in

$$
N^{\prime} \cap p\left\langle M, A \rtimes \Gamma_{0}\right\rangle p \subset p\left(A^{\prime} \cap\left\langle M, A \rtimes \Gamma_{0}\right\rangle\right) p \simeq\left(\mathcal{Z}(A) \bar{\otimes} \ell^{\infty}\left(\Gamma / \Gamma_{0}\right)\right) U_{\sigma} p U_{\sigma}^{*} .
$$

We write $U_{\sigma} d U_{\sigma}^{*}=\left(d_{s}\right)_{s \in \Gamma / \Gamma_{0}}$ as an element in $\mathcal{Z}(A) \bar{\otimes} \ell^{\infty}\left(\Gamma / \Gamma_{0}\right)$. Note that the canonical trace on $\left\langle M, A \rtimes \Gamma_{0}\right\rangle$ is of the form $\operatorname{Tr}_{A \rtimes \Gamma_{0}} \otimes \operatorname{Tr}_{\ell^{2}\left(\Gamma / \Gamma_{0}\right)}$ on $\mathcal{Z}(A) \bar{\otimes} \ell^{\infty}\left(\Gamma / \Gamma_{0}\right)$. When $A$ is a $\mathrm{II}_{1}$ factor and $p=1$, then since $d$ is a trace finite projection, it is contained in $\mathbb{C} \bar{\otimes} \ell^{\infty}(\mathcal{S})$ for some finite $\mathcal{S}$. So we assume $\mathcal{Z}(A)$ is diffuse.

We claim that there exists a projection $e \in \mathcal{Z}(A) p$ such that $U_{\sigma} d e U_{\sigma}^{*}=\left(d_{s} e_{s}\right)_{s} \neq 0$ and $d_{s} e_{s}=0$ except for finitely many $s \in \Gamma / \Gamma_{0}$, where $e_{s}=\alpha_{\tilde{s}}^{-1}(e)$. Let $\psi$ be an isomorphism from $\mathcal{Z}(A) \bar{\otimes} \ell^{\infty}\left(\Gamma / \Gamma_{0}\right)$ onto itself given by $\psi\left(\left(x_{s}\right)_{s}\right)=\left(\alpha_{\tilde{s}}\left(x_{s}\right)\right)_{s}$. Put $\left(\tilde{d}_{s}\right)_{s}:=\psi\left(\left(d_{s}\right)_{s}\right)=\left(\alpha_{\tilde{s}}\left(d_{s}\right)\right)_{s}$. We regard $\left(\operatorname{id}_{\mathcal{Z}(A)} \otimes \operatorname{Tr}_{\ell^{2}\left(\Gamma / \Gamma_{0}\right)}\right)\left(\left(\tilde{d}_{s}\right)_{s}\right)=\sum_{s \in \Gamma / \Gamma_{0}} \tilde{d}_{s}(=:$ $f)$ as a function on the spectrum of $\mathcal{Z}(A)$ which takes values on $[0, \infty]$. Note that $\operatorname{id}_{\mathcal{Z}(A)} \otimes \operatorname{Tr}_{\ell^{2}\left(\Gamma / \Gamma_{0}\right)}$ gives an extended center valued trace on $\mathcal{Z}(A) \bar{\otimes} \mathbb{B}\left(\ell^{2}\left(\Gamma / \Gamma_{0}\right)\right)$. Since $\sum_{s \in \Gamma / \Gamma_{0}} \operatorname{Tr}_{A}\left(\tilde{d}_{s}\right)=\sum_{s \in \Gamma / \Gamma_{0}} \operatorname{Tr}_{A}\left(d_{s}\right)=\operatorname{Tr}_{\left\langle M, A \rtimes \Gamma_{0}\right\rangle}(d)<\infty$, the function $f$ actually takes values on $[0, \infty)$. Let $z$ be a projection in $\mathcal{Z}(A)$ which corresponds to the characteristic function on $f^{-1}([0, n])$ for some large $n \in \mathbb{N}$. Then we have $0 \neq \sum_{s \in \Gamma / \Gamma_{0}} \tilde{d}_{s} z=f z \leq n$ and hence the element $f z$ is contained in $\mathcal{Z}(A)$. Now by Lemma 4.2.1, we can find a projection $w \in \mathcal{Z}(A)$ such that $f z w \neq 0$ and $\tilde{d}_{s} z w=0$ except for finitely many $s \in \Gamma / \Gamma_{0}$. Hence we get $0 \neq \psi^{-1}\left(\left(\tilde{d}_{s} z w\right)_{s}\right)=\left(d_{s} \alpha_{\sigma(s)}^{-1}(z w)\right)_{s}$. Thus $e:=z w$ does the work.

Finally replacing $e \in \mathcal{Z}(A) p$ in the claim with a sufficiently small one, we can assume $d_{s} \alpha_{\tilde{s}}^{-1}(e)=\alpha_{\tilde{s}}^{-1}(e)$ or 0 . Putting $\mathcal{S}:=\left\{s \mid d_{s} \alpha_{\tilde{s}}^{-1}(e)=\alpha_{\tilde{s}}^{-1}(e)\right\}$, we can end the proof.

For finite von Neumann algebras $A \subset M$, we say that the inclusion is a finite extension if for any trace on $A$, the associated semifinite trace on $\langle M, A\rangle$ is finite. We use this notion in the next subsection with the following observation.

Let $B \subset A \subset M$ be finite von Neumann algebras with a fixed trace. We iden-

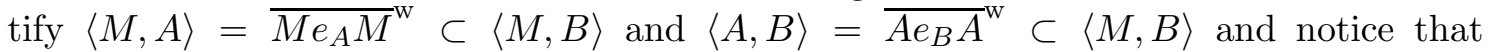
$\left.\operatorname{Tr}_{\langle M, B\rangle}\right|_{\langle A, B\rangle}=\operatorname{Tr}_{\langle A, B\rangle}$. Assume that $B \subset A$ is a finite extension. Then since $\operatorname{Tr}_{\langle M, B\rangle}$ is still semifinite on $\langle M, A\rangle$ (because $\operatorname{Tr}_{\langle M, B\rangle}\left(e_{A}\right)=\operatorname{Tr}_{\langle A, B\rangle}\left(e_{A}\right)<\infty$ ), there is a conditional expectation from $\langle M, B\rangle$ onto $\langle M, A\rangle$.

Remark 4.2.6. Let $\pi$ and $e$ be as in the previous proposition. When $A$ is a $\mathrm{II}_{1}$ factor, $\pi$ is defined on $N$ as a unital and normal one. The inclusion $\pi(A) \subset A \bar{\otimes} \mathbb{B}\left(\ell^{2}(\mathcal{S})\right)$ is a finite extension. When $\mathcal{Z}(A)$ is diffuse, if we further assume $N$ and $A \rtimes \Gamma_{0}$ are $\mathrm{II}_{1}$ factors and $p A p \subset N$ is regular, then we can extend $\pi$ on $N$ in the following way. We first extend $\pi$ on $p A p$ by $\pi(a):=\sum_{s \in \mathcal{S}} \alpha_{\tilde{s}}^{-1}(a) \otimes e_{s, s}$. Exchanging $e$ with a small one, we may assume $e$ has trace $1 / m$ in $N$. Then $\pi(e)=\sum_{s \in \mathcal{S}} \alpha_{\tilde{s}}^{-1}(e) \otimes e_{s, s} \in \pi(p)\left(\mathcal{Z}(A) \bar{\otimes} \mathbb{B}\left(\ell^{2}(\mathcal{S})\right)\right) \pi(p)$ has trace $1 / m$ with $\operatorname{Tr}_{A} \otimes \operatorname{Tr}_{\ell^{2}(\mathcal{S})}$, where $\operatorname{Tr}_{\ell^{2}(\mathcal{S})}$ is the normalized trace. Let $e_{1}:=e$ and $e_{i} \in \mathcal{Z}(A) p$ $(i=2, \ldots, m)$ be mutually orthogonal projections having trace $1 / m$. We have $p=\sum_{i=1}^{m} e_{i}$. By Lemma 4.2.3, take partial isometries $v_{i} \in N$ and $w_{i} \in \pi(p)\left(A \rtimes \Gamma_{0} \bar{\otimes} \mathbb{B}\left(\ell^{2}(\mathcal{S})\right)\right) \pi(p)(i=$ $1, \ldots, m)$ satisfying that $v_{i} v_{i}^{*}=e_{1}, w_{i} w_{i}^{*}=\pi\left(e_{1}\right), v_{i}^{*} v_{i}=e_{i}, w_{i}^{*} w_{i}=\pi\left(e_{i}\right), v_{i} A v_{i}^{*}=A e_{1}$ and $w_{i}\left(A \bar{\otimes} \ell^{\infty}(\mathcal{S})\right) w_{i}^{*}=\left(A \bar{\otimes} \ell^{\infty}(\mathcal{S})\right) \pi\left(e_{1}\right)$. Define $\tilde{\pi}(x):=\sum_{i, j=1}^{m} w_{i}^{*} \pi\left(v_{i} x v_{j}^{*}\right) w_{j}$ for $x \in N$. Then $\tilde{\pi}$ is a unital normal $*$-homomorphism from $N$ into $\pi(p)\left(A \rtimes \Gamma_{0} \bar{\otimes} \mathbb{B}\left(\ell^{2}(\mathcal{S})\right)\right) \pi(p)$ 
satisfying $\tilde{\pi}=\pi$ on $e N e$. Since $\pi(e A e) \subset \pi(e)\left(A \bar{\otimes} \mathbb{B}\left(\ell^{2}(\mathcal{S})\right)\right) \pi(e)$ is a finite extension, $\pi(p A p) \subset \pi(p)\left(A \bar{\otimes} \mathbb{B}\left(\ell^{2}(\mathcal{S})\right)\right) \pi(p)$ is also a finite extension.

Corollary 4.2.7. Assume either that $\left.\alpha\right|_{\Gamma_{0}}$ is ergodic on $\mathcal{Z}(A)$ so that $A \rtimes \Gamma_{0}$ is a $\mathrm{II}_{1}$ factor, or $\alpha$ is free on $\mathcal{Z}(A)$. If $N \preceq_{M} A \rtimes \Gamma_{0}$, then there exist non-zero projections e, $f \in \mathcal{Z}(A)$ (or $e, f \in A$ when $A$ is a $\mathrm{II}_{1}$ factor) with $e \leq p$, a normal unital $*$-homomorphism $\theta: e N e \rightarrow$ $f\left(A \rtimes \Gamma_{0}\right) f$, and a non-zero partial isometry $v \in e(A \rtimes \Gamma) f$ such that $x v=v \theta(x)$ for $x \in e N e, v v^{*} \in \mathcal{Z}(A) e \cap N^{\prime}$, and $\theta(e A e) \subset f A f$. In the case, the inclusion $\theta(e A e) \subset f A f$ is a finite extension.

Proof. Let $e, d, \mathcal{S}$, and $\pi$ be as in the previous proposition. Write $\mathcal{S}=\left\{s_{1}, \ldots, s_{n}\right\}$ and write the matrix unit of $\mathbb{B}\left(\ell^{2}(\mathcal{S})\right)$ along $s_{i}$ as $\left(e_{i, j}\right)_{i, j}$. Assume first $\mathcal{Z}(A)$ is diffuse and $A \rtimes \Gamma_{0}$ is a $\mathrm{II}_{1}$ factor. Then there are projections $e_{i} \in \mathcal{Z}(A)$ such that $e=\sum_{i=1}^{n} e_{i}$ and each $e_{i}$ has the same trace in $A \rtimes \Gamma_{0}$. We may assume $d e_{1} \neq 0$ so that $\pi\left(e_{1}\right) \neq 0$. By the proof of Corollary 2.3.7 (regarding $e_{1} N e_{1} \simeq e_{1} N e_{1} \bar{\otimes} \mathbb{C} e_{1,1}$ ), there is a partial isometry $w \in\left(e_{1} \otimes e_{1,1}\right)\left(M \bar{\otimes} \mathbb{M}_{n}\right)$ such that $\left(x \otimes e_{1,1}\right) w=w \pi(x)$ for any $x \in e_{1} N e_{1}$. This equation implies $a w_{j}=w_{j} \alpha_{\tilde{s}_{j}}^{-1}(a)$ for all $a \in A$, where we write $w=\sum_{j} w_{j} \otimes e_{1, j}$. By a similar manner to that in the proof of the last lemma, we get $w_{j}=a_{j} \lambda_{\tilde{s}_{j}}$ for some $a_{j} \in e_{1} \mathcal{Z}(A)$. By Lemma 4.2.3, take partial isometries $u_{i}$ in $A \rtimes \Gamma_{0}$ such that $u_{i}^{*} u_{i}=e_{i}, u_{i} u_{i}^{*}=\alpha_{\tilde{s}_{i}}^{-1}\left(e_{1}\right)$, and $u_{i}^{*} A u_{i}=e_{i} A$. Put $U:=\left(\delta_{i, j} u_{i}\right)_{i, j}$. Let $V$ be a partial isometry in $\left(A \rtimes \Gamma_{0}\right) \bar{\otimes} \mathbb{B}\left(\ell^{2}(\mathcal{S})\right)$ given by $V_{i, j}=e_{i} \delta_{1, j}$ and note $V V^{*}=U^{*} \pi\left(e_{1}\right) U$. Put $\theta:=\operatorname{Ad}\left(V^{*} U^{*}\right) \circ \pi: e_{1} N e_{1} \rightarrow A \rtimes \Gamma_{0} \bar{\otimes} \mathbb{C} e_{1,1} \simeq A \rtimes \Gamma_{0}, \theta\left(e_{1}\right)=\sum_{i=1}^{n} e_{i}=: f$ and $w U V \simeq \sum_{i} a_{i} \lambda_{\tilde{s}_{i}} u_{i}=: v$. We have $v \theta(x)=x v$ for $x \in e_{1} N e_{1}$ and hence $v v^{*} \in\left(e_{1} N e_{1}\right)^{\prime}$. Also we have $v v^{*}=\sum_{i} a_{i} \lambda_{\tilde{s}_{i}} u_{i} u_{i}^{*} \lambda_{\tilde{s}_{i}}^{*} a_{i}^{*}=\sum_{i} a_{i} \lambda_{\tilde{s}_{i}} \alpha_{\tilde{s}_{i}}^{-1}\left(e_{1}\right) \lambda_{\tilde{s}_{i}}^{*} a_{i}^{*}=\sum_{i} a_{i} a_{i}^{*} \in e_{1} \mathcal{Z}(A)$ and $\theta(a)=\sum_{i=1}^{n} u_{i}^{*} \alpha_{\tilde{s}}^{-1}(a) u_{i} \in f A f$ for $a \in A e_{1}$. Finally $\theta\left(e_{1} A e_{1}\right) \subset f A f$ is a finite extension, since so is $\operatorname{Ad} U^{*} \circ \pi\left(e_{1} A e_{1}\right) \subset E\left(A \bar{\otimes} \mathbb{B}\left(\ell^{2}(\mathcal{S})\right)\right) E$, where $E:=\operatorname{Ad} U^{*} \circ \pi\left(e_{1}\right)$.

Next assume that $A$ is a $\mathrm{II}_{1}$ factor so that $\operatorname{Tr}_{A}$ is finite and $p=1$. Then decomposing $e=1_{A}=\sum_{i=1}^{n} e_{i}$ for $e_{i} \in A$ with $e_{i} \sim e_{j}$ in $A$, we can take $u_{i}, U, V, v, \pi$, and $\theta$ as above, which do the work.

Finally assume $\mathcal{Z}(A)$ is diffuse and $\alpha$ is free on $\mathcal{Z}(A)$. By Lemma 4.2.2, there is $e_{1} \leq e$ such that $\alpha_{\tilde{s}}^{-1}\left(e_{1}\right) \alpha_{\tilde{t}}^{-1}\left(e_{1}\right)=0$ for any $s, t \in \mathcal{S}$ with $s \neq t$. In the case, we do not need to take $u_{i}$ above. So putting $\theta:=\left.\operatorname{Ad} V^{*} \circ \pi\right|_{e_{1} N e_{1}}$, we are done.

\subsection{Proof of Theorem B}

In the subsection, we use the same notation as in Theorem $\mathrm{B}$, We write $M:=A \rtimes \Gamma$, $\Gamma_{X}:=\prod_{i \in X} \Gamma_{i}$ and $\Lambda_{Y}:=\prod_{j \in Y} \Lambda_{j}$ for $Y \subset\{1, \ldots, n\}$ and $X \subset\{1, \ldots, m\}$.

Lemma 4.3.1. For any subset $Y \subset\{1, \ldots, n\}$ with $|Y| \leq m$, there is $X \subset\{1, \ldots, n\}$ such that $|X|=|Y|$ and $B \rtimes \Lambda_{Y} \preceq_{M} A \rtimes \Gamma_{X}$.

Proof. We apply Proposition 4.1.2 to $B \rtimes\left(\Lambda_{2} \times \cdots \times \Lambda_{n}\right) \subset p M p$. If there is an expectation from $p\langle M, A\rangle p$ onto $L\left(\Lambda_{2} \times \cdots \times \Lambda_{n}\right)^{\prime}$ which contains $L \Lambda_{1}$, then we have a contradiction when $A$ is amenable because $\langle M, A\rangle$ is amenable. When $A$ is non-amenable and $p=1$, we have an expectation from $\mathbb{C} 1_{B} \bar{\otimes} \mathbb{B}\left(\ell^{2}(\Lambda)\right) \subset\langle B \rtimes \Lambda, B\rangle \subset\langle M, A\rangle$ into $L \Lambda_{1}$ and hence a contradiction. So we have $B \rtimes\left(\Lambda_{2} \times \cdots \times \Lambda_{n}\right) \preceq_{M} A \rtimes \Gamma_{X_{1}}$ for some $X_{1}:=\{1, \ldots, m\} \backslash\{i\}$. For simplicity we assume $i=1$.

We take $e, d, \mathcal{S}$, and $\pi$ in Proposition 4.2.5 and write $\mathbb{B}\left(\ell^{2}(\mathcal{S})\right)=: \mathbb{M}_{m_{1}}$ and $\tilde{A}:=A \bar{\otimes}$ $\mathbb{M}_{m_{1}}$. Considering the trivial action of $\Gamma_{X_{1}}$ on $\mathbb{M}_{m_{1}}$, we regard $\left(A \rtimes \Gamma_{X_{1}}\right) \bar{\otimes} \mathbb{M}_{m_{1}}=\tilde{A} \rtimes \Gamma_{X_{1}}$. Here we claim that $\pi\left(e\left(B \rtimes\left(\Lambda_{3} \times \cdots \times \Lambda_{n}\right)\right) e\right) \preceq_{\pi(e)\left(\tilde{A} \rtimes \Gamma_{X_{1}}\right) \pi(e)} \pi(e)\left(\tilde{A} \rtimes \Gamma_{X_{2}}\right) \pi(e)$ for some $X_{2}:=X_{1} \backslash\{i\}$ (we assume $i=2$ for simplicity). By Remark 4.2.6, we extend $\pi$ on $B \rtimes\left(\Lambda_{2} \times \cdots \times \Lambda_{n}\right)$ and apply again Proposition 4.1.2 to $\pi\left(B \rtimes\left(\Lambda_{3} \times \cdots \times \Lambda_{n}\right)\right) \subset \tilde{A} \rtimes \Gamma_{X_{1}}$. If 
there is an expectation from $\pi(p)\left\langle\tilde{A} \rtimes \Gamma_{X_{1}}, \tilde{A}\right\rangle \pi(p)$ onto $\pi\left(L\left(\Lambda_{3} \times \cdots \times \Lambda_{n}\right)\right)^{\prime}$ which contains $\pi\left(L \Lambda_{2}\right)$, then we have a contradiction when $A$ is amenable. When $A$ is non-amenable and $p=1$, since $\pi(B) \subset \tilde{A}$ is a finite extension, there is an expectation from $\left\langle\tilde{A} \rtimes \Gamma_{X_{1}}, \pi(B)\right\rangle$ onto $\left\langle\tilde{A} \rtimes \Gamma_{X_{1}}, \tilde{A}\right\rangle$. So we have an expectation from $\left\langle\pi\left(B \rtimes\left(\Lambda_{2} \times \cdots \times \Lambda_{n}\right)\right), \pi(B)\right\rangle$, which is a subalgebras of $\left\langle\tilde{A} \rtimes \Gamma_{X_{1}}, \pi(B)\right\rangle$, onto $\pi\left(L \Lambda_{2}\right)$. This contradicts to the amenability of $\Lambda_{2}$. Thus we get $\pi\left(B \rtimes\left(\Lambda_{3} \times \cdots \times \Lambda_{n}\right)\right) \preceq_{\tilde{A} \rtimes \Gamma_{X_{1}}} \tilde{A} \rtimes \Gamma_{X_{2}}$ for some $X_{2}$. By Corollary 2.3.6, we get the claim.

Now by construction, we are in fact seeing $e\left(B \rtimes\left(\Lambda_{2} \times \cdots \times \Lambda_{n}\right)\right) e d \subset \pi(e)\left(A \rtimes \Gamma_{X_{1}} \bar{\otimes}\right.$ $\left.\mathbb{B}\left(\ell^{2}(\mathcal{S})\right)\right) \pi(e)$. So by the same manner as in the proof of Lemma 3.4.3, we can deduce $B \rtimes\left(\Lambda_{3} \times \cdots \times \Lambda_{n}\right) \preceq_{M} A \rtimes \Gamma_{X_{2}}$. This completes the proof.

Proof of Theorem B (first half). Suppose by contradiction that $n>m$. Then by the previous lemma, we have $B \rtimes \Lambda_{Y} \preceq_{M} A$ for some $Y \neq \emptyset$. By Proposition 4.2.5 and Remark 4.2.6, we have a $*$-homomorphism $\pi: B \rtimes \Lambda_{Y} \rightarrow A \bar{\otimes} \mathbb{M}_{n}$ for some $n$ such that $\pi(B) \subset \pi(p)\left(A \bar{\otimes} \mathbb{M}_{n}\right) \pi(p)$ is a finite extension. Then since $\pi(B)$ is co-amenable in $\pi(p)\left(A \bar{\otimes} \mathbb{M}_{n}\right) \pi(p), \pi(B)$ is co-amenable in $\pi\left(B \rtimes \Lambda_{Y}\right)$. This contradicts to the nonamenability of $\Lambda_{Y}$.

By Lemma 4.3.1, if $n=m$, for any $i$ there is some $j$ such that $B \rtimes \Lambda_{i} \preceq_{M} A \rtimes \Gamma_{j}$. We show that the assignment $i \mapsto j$ is one to one.

Lemma 4.3.2. If $B \rtimes \Lambda_{i} \preceq_{M} A \rtimes \Gamma_{j}$ and $B \rtimes \Lambda_{i} \preceq_{M} A \rtimes \Gamma_{l}$ for some $i, j$ and $l$, then we have $j=l$.

Proof. Suppose $j \neq l$. Since $\left(B \rtimes \Lambda_{i}\right)^{\prime} \cap p M p=\mathbb{C} p$, by Lemma 2.4.6, we have $B \rtimes$ $\Lambda_{i} \subset_{\text {approx }} A \rtimes \Gamma_{a}$ for $a=j, l$. Hence we have $B \rtimes \Lambda_{i} \subset_{\text {approx }} A$ by Lemma 2.4.8. This implies $B \rtimes \Lambda_{i} \preceq_{M} A$ and it contradicts to (the proof of) the first part of Theorem $\mathrm{B}$,

The proof of the following lemma was from that of [21, Lemma 33].

Lemma 4.3.3. If $B \rtimes \Lambda_{i} \preceq_{M} A \rtimes \Gamma_{j}$ and $B \rtimes \Lambda_{k} \preceq_{M} A \rtimes \Gamma_{j}$ for some $i, j$ and $k$, then we have $i=k$.

Proof. By assumption, there are non-zero trace finite projections $e_{a} \in\left(B \rtimes \Lambda_{a}\right)^{\prime} \cap((A \rtimes$ $\left.\left.\Gamma_{j}\right)^{\mathrm{op}}\right)^{\prime}$ with $e_{a}=e_{a} p$ for $a=i, k$. Observe that for any $s \in \Lambda_{\{i\}^{c}}$ and $g \in \Gamma_{\{j\}^{c}}$, the element $\rho_{g} \lambda_{s} e_{i} \lambda_{s}^{*} \rho_{g}^{*}$ satisfies the same condition as that on $e_{i}$. Let $e$ be the element $\sup _{s \in \Lambda_{\{i\} c}, g \in \Gamma_{\{j\}^{c}}} \rho_{g} \lambda_{s} e_{i} \lambda_{s}^{*} \rho_{g}^{*}$. Then $e$ is contained in

$$
\begin{aligned}
& \left(L \Lambda_{\{i\}^{c}}\right)^{\prime} \cap\left(B \rtimes \Lambda_{i}\right)^{\prime} p \cap\left(\left(L \Gamma_{\{j\}^{c}}\right)^{\mathrm{op}}\right)^{\prime} \cap\left(\left(A \rtimes \Gamma_{j}\right)^{\mathrm{op}}\right)^{\prime} \\
= & (B \rtimes \Lambda)^{\prime} p \cap\left((A \rtimes \Gamma)^{\mathrm{op}}\right)^{\prime} \\
= & (B \rtimes \Lambda)^{\prime} p \cap(A \rtimes \Gamma) \\
= & (B \rtimes \Lambda)^{\prime} \cap p \mathcal{Z}(A)=L \Lambda^{\prime} \cap \mathcal{Z}(B)=\mathbb{C} p .
\end{aligned}
$$

Hence we have $e=p$. Thus there exist finite subsets $\mathcal{E} \subset \Lambda_{\{i\}^{c}}$ and $\mathcal{F} \subset \Gamma_{\{j\}^{c}}$ satisfying that $\vee_{s \in \mathcal{E}, g \in \mathcal{F}} \rho_{g} \lambda_{s} e_{i} \lambda_{s}^{*} \rho_{g}^{*}$ is not orthogonal to $e_{k}$. By exchanging $e_{i}$ with this element, we can assume $e_{i} e_{k} \neq 0$.

Suppose now $i \neq k$. We claim that $B \rtimes\left(\Lambda_{i} \times \Lambda_{k}\right) \preceq_{M} A \rtimes \Gamma_{j}$. Consider the $\sigma$-weak closure of $\operatorname{co}\left\{\lambda_{s} e_{i} \lambda_{s}^{*} \mid s \in \Lambda_{k}\right\}$ and, regarding this set as a subset of $L^{2}\left(\left\langle A \rtimes \Gamma, A \rtimes \Gamma_{j}\right\rangle\right)$, take the circumcenter $d$, which is contained in $L \Lambda_{k}^{\prime} \cap\left(B \rtimes \Lambda_{i}\right)^{\prime} p \cap\left(\left(A \rtimes \Gamma_{j}\right)^{\mathrm{op}}\right)^{\prime}=(B \rtimes$ $\left.\left(\Lambda_{i} \times \Lambda_{k}\right)\right)^{\prime} p \cap\left(\left(A \rtimes \Gamma_{j}\right)^{\text {op }}\right)^{\prime}$. This is non-zero since we have for any $s \in \Lambda_{k}$,

$$
\left\langle\lambda_{s} e_{i} \lambda_{s}^{*}, e_{k}\right\rangle=\operatorname{Tr}_{\left\langle A \rtimes \Gamma, A \rtimes \Gamma_{i}\right\rangle}\left(\lambda_{s} e_{i} \lambda_{s}^{*} e_{k}\right)=\operatorname{Tr}_{\left\langle A \rtimes \Gamma, A \rtimes \Gamma_{i}\right\rangle}\left(e_{i} e_{k}\right)>0 .
$$


So we get the claim.

Now by the proof of Lemma 4.3.1, we have $B \rtimes \Lambda_{i} \preceq_{M} A$. This contradicts to (the proof of) the first part of Theorem $B$.

Thanks for previous two lemmas, the assignment $i \mapsto j$ above gives a bijective map on $\{1, \ldots, n\}$. Putting $j=\sigma(i)$, we complete the proof.

\section{Another approach to prime factorization results}

\subsection{Irreducibility and primeness}

In the number theory, there are two notions of prime numbers. Recall that a number $p \in \mathbb{N}$ is irreducible if for any $q, r \in \mathbb{N}$ with $p=q r$, we have $q=1$ or $r=1$; and is prime if for any $q, r, s \in \mathbb{N}$ with $p q=r s$, we have $p \mid r$ or $p \mid s$. In our von Neumann algebra theory, we used irreducibility as a definition of primeness for von Neumann algebras. If we adopt primeness of the number theory, the following condition should be a corresponding notion:

- We say a $\mathrm{II}_{1}$ factor $M$ is "prime" if for any $\mathrm{II}_{1}$ factor $N, K, L$ with $M \bar{\otimes} N=K \bar{\otimes} L$, there is a unitary $u \in \mathcal{U}(M)$ and $t>0$ such that $u M u^{*} \subset K^{t}$ or $u M u^{*} \subset L^{t}$.

We prove that there are such examples.

Theorem 5.1.1. Let $\Gamma$ be a discrete group. Assume that $\Gamma$ is non-amenable, ICC, bi-exact and weakly amenable. Then for any $\mathrm{II}_{1}$ factor $B, K$ and $L$ with $L \Gamma \bar{\otimes} B=K \bar{\otimes} L(=: M)$, we have either $L \Gamma \preceq_{M} K$ or $L \Gamma \preceq_{M} L$. If $L \Gamma \preceq_{M} K$, then there is a unitary $u \in \mathcal{U}(M)$ and $t>0$ such that $u L \Gamma u^{*} \subset K^{t}$.

Proof. Suppose by contradiction that $L \Gamma \npreceq_{M} K$ and $L \Gamma \npreceq_{M} L$. By [26, Lemma 3.5], this exactly means $K \npreceq_{M} B$ and $L \swarrow_{M} B$. By [2, Corollary F.14], there is a diffuse abelian subalgebra $A \subset K$ such that $A \npreceq_{M} B$. By [20, Theorem 1.4], $\mathcal{N}_{M}(A)^{\prime \prime}$ is amenable relative to $B$ in M. Since $L \subset \mathcal{N}_{M}(A)^{\prime \prime}, L$ is also amenable relative to $B$. We again apply [20, Theorem 1.4] to $L$ and get that $M=\mathcal{N}_{M}(L)^{\prime \prime}$ is amenable relative to $B$. This means $L \Gamma$ is amenable and hence a contradiction. The last assertion follows from Lemma 3.3.1.

Remark 5.1.2. Since we generalized [20, Theorem 1.4] to quantum groups of Kac type [12, Theorem A], the same thing is true for $\mathrm{II}_{1}$ factors of $L^{\infty}\left(\mathbb{G}_{i}\right)$, where $\hat{\mathbb{G}}_{i}$ is non-amenable, bi-exact and weakly amenable.

Once we get the property, it is easy to deduce the following prime factorization results. Since proofs are straightforward, we leave it to the reader.

Corollary 5.1.3. Let $M_{i}(i=1, \ldots, m)$ be $\mathrm{II}_{1}$ factors. Assume that each $M_{i}$ is "prime" in the above sense. Let $M_{0}$ and $N_{j}(j=0,1, \ldots, n)$ be prime $\mathrm{II}_{1}$ factors in the usual sense satisfying $M_{0} \bar{\otimes} M_{1} \bar{\otimes} \cdots \bar{\otimes} M_{m}=N_{0} \bar{\otimes} \cdots \bar{\otimes} N_{n}(=: M)$. Then $n=m$ and there are $a$ unitary $u \in \mathcal{U}(M), \sigma \in \mathfrak{S}_{n+1}$, and $t_{i}>0$ with $t_{0} \cdots t_{n}=1$ such that $u M_{i} u^{*}=N_{\sigma(i)}^{t_{i}}$.

Corollary 5.1.4. Let $M_{i}(i=1, \ldots, m), M_{0}$, and $M$ be as in the previous corollary. Then $\mathcal{F}(M)=\mathcal{F}\left(M_{0}\right) \mathcal{F}\left(M_{1}\right) \cdots \mathcal{F}\left(M_{m}\right)$. Here $\mathcal{F}(M)$ and $\mathcal{F}\left(M_{i}\right)$ are fundamental groups of $M$ and $M_{i}$. 


\section{Reference}

[1] R. Boutonnet, C. Houdayer, and S. Raum, Amalgamated free product type III factors with at most one Cartan subalgebras, Compos. Math. 150 (2014), 143-174.

[2] N. P. Brown and N. Ozawa, $C^{*}$-algebras and finite-dimensional approximations. Graduate Studies in Mathematics, 88. American Mathematical Society, Providence, RI, 2008.

[3] I. Chifan and C. Houdayer, Bass-Serre rigidity results in von Neumann algebras, Duke Math. J. 153 (2010), 23-54.

[4] A. Connes, Almost periodic states and factors of type $\mathrm{III}_{1}$, J. Funct. Anal. 16 (1974), 415-445.

[5] A. Connes, Classification of injective factors, Ann. of Math. (2) 104 (1976), 73-115.

[6] K. Dykema, Crossed product decompositions of a purely infinite von Neumann algebra with faithful, almost periodic weight, Indiana Univ. Math. J. 44 (1995), 433-450.

[7] L. Ge, Applications of free entropy to finite von Neumann algebras. II, Ann. of Math. (2) 147 (1998), no. 1, 143-157.

[8] C. Houdayer and E. Ricard, Approximation properties and absence of Cartan subalgebra for free Araki-Woods factors, Adv. Math. 228 (2011), 764-802.

[9] C. Houdayer and S. Vaes, Type III factors with unique Cartan decomposition, J. Math. Pures Appl. 100 (2013), 564-590.

[10] Y. Isono, Examples of factors which have no Cartan subalgebras, to appear in Trans. Amer. Math. Soc.

[11] Y. Isono, Weak Exactness for $C^{*}$-algebras and Application to Condition (AO), J. Funct. Anal. 264 (2013), 964-998.

[12] Y. Isono, On bi-exactness of discrete quantum groups, to appear in Int. Math. Res. Not.

[13] N. Ozawa, A Kurosh type theorem for type $\mathrm{II}_{1}$ factors. Int. Math. Res. Not. (2006), Art. ID 97560, 21 pp.

[14] N. Ozawa, Solid von Neumann algebras. Acta Math. 192 (2004), 111-117.

[15] N. Ozawa and S. Popa, Some prime factorization results for type $\mathrm{II}_{1}$ factors, Invent. Math. 156 (2004), 223-234.

[16] J. Peterson, $L^{2}$-rigidity in von Neumann algebras, Invent. Math. 175 (2009), 417-433.

[17] S. Popa, Strong rigidity of $\mathrm{II}_{1}$ factors arising from malleable actions of w-rigid groups I, Invent. Math. 165 (2006), 369-408.

[18] S. Popa, On a class of type $\mathrm{II}_{1}$ factors with Betti numbers invariants, Ann. of Math. 163 (2006), 809-899.

[19] S. Popa, Orthogonal pairs of *-subalgebras in finite von Neumann algebras, J. Op. Theory 9 (1983), 253-268. 
[20] S. Popa and S. Vaes, Unique Cartan decomposition for $\mathrm{II}_{1}$ factors arising from arbitrary actions of hyperbolic groups, to appear in J. Reine Angew. Math.

[21] H. Sako, Measure equivalence rigidity and bi-exactness of groups, J. Funct. Anal. 257 (10) 3167-3202, 2009.

[22] M. Takesaki, Theory of operator algebras I, Encyclopedia of Mathematical Sciences, 124. Operator Algebras and Non-commutative Geometry, 5. Springer-Verlag, Berlin, 2002 .

[23] M. Takesaki, Theory of operator algebras II, Encyclopedia of Mathematical Sciences, 125. Operator Algebras and Non-commutative Geometry, 5. Springer-Verlag, Berlin, 2002.

[24] M. Takesaki, Theory of operator algebras III, Encyclopedia of Mathematical Sciences, 127. Operator Algebras and Non-commutative Geometry, 5. Springer-Verlag, Berlin, 2003.

[25] Y. Ueda, Some analysis on amalgamated free products of von Neumann algebras in non-tracial setting, J. London Math. Soc. 88 (2013), no.1, 25-48.

[26] S. Vaes, Explicit computations of all finite index bimodules for a family of $\mathrm{II}_{1}$ factors, Ann. Sci. Ecole Norm. Sup. 41 (2008), 743-788.

[27] S. Vaes, One-cohomology and the uniqueness of the group measure space decomposition of a II $_{1}$ factor, Math. Ann. 355 (2013), 661-696.

[28] R. Vergnioux, Orientation of quantum Cayley trees and applications, J. Reine Angew. Math. 580 (2005), 101-138.

[29] S. Vaes and N. Vander Vennet, Poisson boundary of the discrete quantum group $\widehat{A_{u}(F)}$, Compos. Math. 146 (4) (2010), 1073-1095.

[30] S. Vaes and R. Vergnioux, The boundary of universal discrete quantum groups, exactness, and factoriality, Duke Math. J. 140 (2007), 35-84.

[31] A. Van Daele and S. Wang, Universal quantum groups, Internat. J. Math. 7 (1996), $255-263$.

[32] S. Wang, Free products of compact quantum groups, Comm. Math. Phys. 167 (1995), no.3, 671-692. 